\title{
Resting-State Activity in High-Order Visual Areas as a Window into Natural Human Brain Activations
}

\author{
Francesca Strappini ${ }^{1}$, Meytal Wilf2,3, Ofer Karp², Hagar Goldberg², \\ Michal Harel2 ${ }^{2}$ Edna Furman-Haran ${ }^{4}$, Tal Golan ${ }^{5}$ and Rafael Malach ${ }^{2}$
}

${ }^{1}$ Istituto Neurologico Mediterraneo IRCCS NEUROMED, Pozzilli 86077, Italy, ${ }^{2}$ Neurobiology Department, Weizmann Institute of Science, Rehovot 76100, Israel, ${ }^{3}$ Department of Clinical Neurosciences, MySpace Lab, Lausanne University Hospital, Lausanne 1011, Switzerland, 'Life Sciences Core Facilities Department, Weizmann Institute of Science, Rehovot 76100, Israel and ${ }^{5}$ The Edmund and Lily Safra Center for Brain Sciences, Hebrew University of Jerusalem, Jerusalem 9190401, Israel

Address correspondence to Rafael Malach, Neurobiology Department, Weizmann Institute of Science, Herzl St 234,7610001 Rehovot, Israel. Email: rafi.malach@gmail.com

F. Strappini and M. Wilf contributed equally to this work

\begin{abstract}
A major limitation of conventional human brain research has been its basis in highly artificial laboratory experiments. Due to technical constraints, little is known about the nature of cortical activations during ecological real life. We have previously proposed the "spontaneous trait reactivation (STR)" hypothesis arguing that resting-state patterns, which emerge spontaneously in the absence of external stimulus, reflect the statistics of habitual cortical activations during real life. Therefore, these patterns can serve as a window into daily life cortical activity. A straightforward prediction of this hypothesis is that spontaneous patterns should preferentially correlate to patterns generated by naturalistic stimuli compared with artificial ones. Here we targeted high-level category-selective visual areas and tested this prediction by comparing BOLD functional connectivity patterns formed during rest to patterns formed in response to naturalistic stimuli, as well as to more artificial category-selective, dynamic stimuli. Our results revealed a significant correlation between the resting-state patterns and functional connectivity patterns generated by naturalistic stimuli. Furthermore, the correlations to naturalistic stimuli were significantly higher than those found between resting-state patterns and those generated by artificial control stimuli. These findings provide evidence of a stringent link between spontaneous patterns and the activation patterns during natural vision.
\end{abstract}

Key words: free viewing, movie, ventral occipital-temporal cortex, naturalistic scenes, visual cortex

\section{Introduction}

The cerebral cortex is characterized by spontaneous ultraslow fluctuations $(\sim 0.01-0.1 \mathrm{~Hz})$ in neuronal activity within widely distributed cortical regions. These fluctuations, usually studied during a state of rest, are termed "resting-state fluctuations" (Fox and Raichle 2007). Regions with highly correlated restingstate fluctuations are termed "functionally connected" (Biswal et al., 1995). While resting-state functional connectivity patterns have been found to show significant similarities with large-scale networks activated during various tasks (Greicius and Menon 2004; Smith et al., 2009; Cole et al., 2016), the source of their specific organization has not been fully elucidated. We and others have recently proposed that in addition to highlighting large-scale anatomical networks, the detailed connectivity 
structure of resting-state patterns may contain meaningful information about cortical function (Arieli et al., 1996; Tsodyks et al., 1999; Kenet et al., 2003; Fox et al., 2006; Lewis et al., 2009; Snyder and Raichle 2012; Harmelech et al., 2013; Sadaghiani and Kleinschmidt 2013). We have proposed "the spontaneous trait reactivation" (STR) hypothesis (Harmelech and Malach 2013), arguing that neuronal coactivations, which occur habitually during the ecological life of the individual, are spontaneously revealed during rest. If this hypothesis is true, it raises the exciting possibility that spontaneous resting-state patterns offer a unique "window" into the topography of brain coactivations during everyday life. Such a window is of extreme importance, since a major limitation of current human brain research, particularly in the visual domain, is strict dependence on tightly confined, artificial laboratory conditions. Indeed, a large body of research has shown that natural stimuli can reveal neural properties which do not manifest when using simple, parametric stimuli (cf. Review of Felsen and Dan 2005). Several studies seem to be compatible with the STR hypothesis, with findings such as similarities between spontaneous activity and natural scene-evoked activity during visual cortex development (Berkes et al., 2011), evidence of task-activation traces in resting-state patterns (Tambini et al., 2010; Shibata et al., 2011; Harmelech et al., 2013), changes in resting-state patterns after specific training such as perceptual learning (Lewis et al., 2009), and unique patterns associated with individual brains (Finn et al., 2015; Wang et al., 2015; Tavor et al., 2016). A straightforward prediction of the STR hypothesis is that the spontaneous patterns should bear more resemblance to patterns produced under naturalistic as compared with artificial conditions. Recently, we examined the validity of this prediction in retinotopically defined V1, V2, and V3 areas in the human early visual cortex (Wilf et al., 2015), revealing significant similarities between spontaneous and movie-driven patterns. However, a critical question regarding the source of this correlation remains open. One could consider two alternative models to explain spontaneous activity patterns in the early visual cortex: one model suggests that the spontaneous patterns reflect merely the low-level optical statistics of the natural world, while the alternative model proposes that the spontaneous patterns reflect the network coactivation statistics while experiencing the natural world. As early retinotopic visual areas are strongly related to the optical statistics of the visual world, it is difficult to disentangle the two options in these areas. However, in contrast, higher order visual areas show a striking shift from low-level optical selectivity to higher level, gestalt-like properties (although some retinotopic bias remains (Levy et al., 2001)). Higher order visual areas are dominated by gestalt properties and categorical preferences that go beyond the low-level feature sensitivity (Kanwisher et al., 1997; Tong et al., 1998; Grill-Spector and Malach 2004). Furthermore, a clear departure from low-level stimulation has been demonstrated in the insensitivity of higher order visual areas to optical disruptions due to oculomotor activity (Golan et al., 2016, 2017). Thus, a large body of research emphasizes the dominance of categorical information rather than low-order optical stimulation as the central driving force in high-order visual representations. By examining spontaneous patterns in these areas, we could directly test the generality of the hypothesis that spontaneous patterns reflect habitual neuronal coactivations, even when the statistics of the optical stimuli are not directly represented in the network activations.

While boundaries between early visual areas rely on visual field maps, parcellation of the extrastriate category-selective cortex has generally been based on activation preference for a particular visual category (e.g., faces, houses, tools). However, this mapping approach has some limitations. For instance, adjacent subareas with different functional preferences might be bound together in the same area if they show selectivity for a general category (e.g., they all respond to houses). Alternatively, it has been proposed that the organization of the category-selective cortex may be further constrained by the functional connections among areas with the same activation preferences (Mahon and Caramazza 2011; Stevens et al., 2015) and between these areas and the rest of the brain, thus extending the category-selective networks even beyond the visual cortex (Hutchison et al., 2014). Moreover, recent studies have shown that visual categories, such as faces and words, are more closely related to distributed neural networks than to specific regions (Behrmann and Plaut 2013). Given the complexity of these representations, we opted not to subdivide the category-selective cortex. Instead, we examined the possibility of a unifying principle that might account for the complex connectivity patterns within the higher order visual cortex, namely, that patterns are driven by the statistics of habitual coactivations in response to naturalistic stimuli.

\section{Materials and Methods}

\section{Participants}

The participants were 16 healthy adults (mean age 28, range 24-32, 8 females, all right handed) with normal or corrected-tonormal visual acuity and no history of psychiatric, neurological, or attentional disorders. One subject was discarded due to a considerable artifact in functional MRI signals.

The experimental procedure included multiple fMRI sessions per participant. In aggregate, these sessions included four visual conditions, four spontaneous resting-state scans (two resting-state runs with eyes-closed [EC] and two resting-state runs with eyes-open [EO]), and a set of anatomical scans (Fig. 1A). Each condition was repeated twice within a session. The order of conditions was counterbalanced across participants with the exception that the first session always began with a resting-state run.

\section{Visual Stimuli and Experimental Paradigms}

\section{Resting State}

Under the main resting-state condition, 15 participants were instructed to close their eyes and rest for two runs of $8 \mathrm{~min}$ each. In addition, as a control experiment, 12 out of the 15 participants were instructed to fixate on a cross presented in the middle of the screen for two 8-min runs. For both resting-state conditions, subjects were instructed to rest without thinking about anything particular

\section{Movie and Scrambled-Movie Viewing}

Fifteen participants freely viewed two runs of an 8-min segment of a naturalistic feature film in Hebrew [Nir Bergman's Broken Wings (2002)]. The movie segment contained several highly emotional scenes, which included people conversing and using tools, music, and landscape shots. The movie segment was preceded by $15 \mathrm{~s}$ of a blank screen and a 15-s visual adaptation stimulus (alternating colorful patterns). Only the 8min movie segment was used for the analyses.

Furthermore, 14 out of the 15 participants viewed two runs of the same 8-min segment which was phase-scrambled in the space domain. This scrambling procedure was meant to 


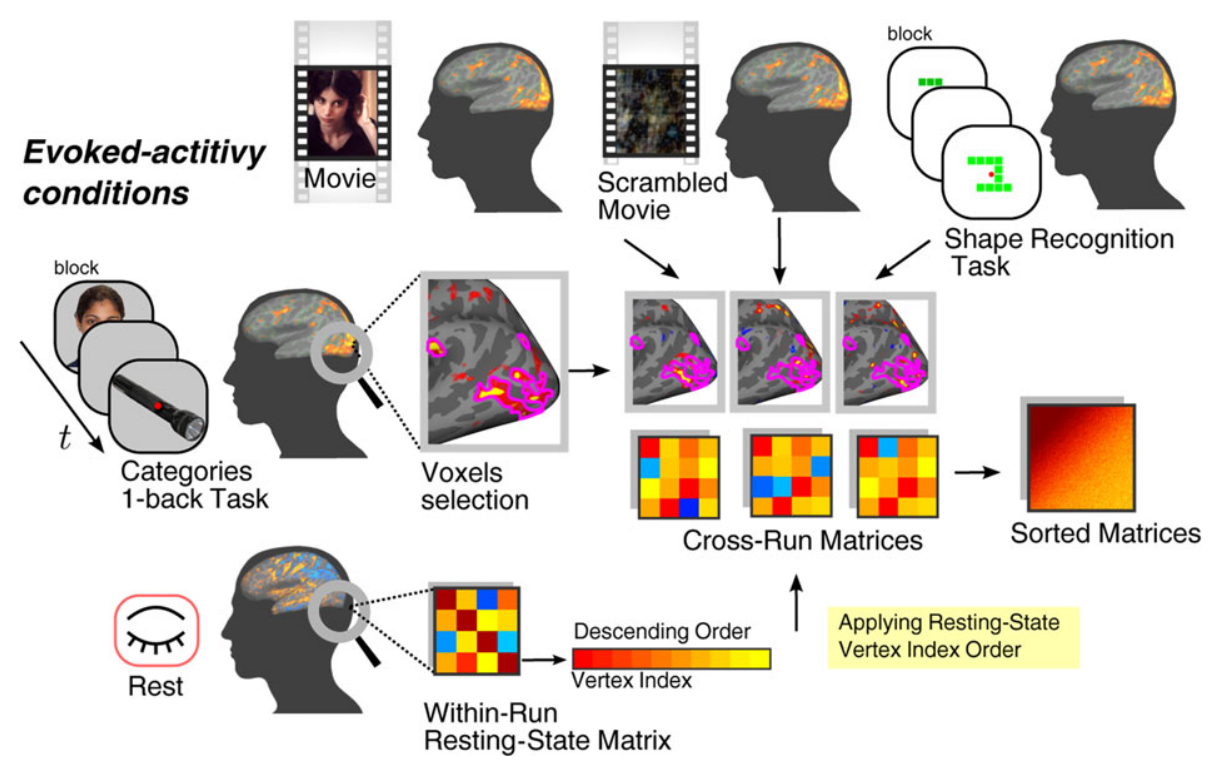

Figure 1. Definition of the region of interest and voxelwise correlation matrices. The figure schematically shows each step of the processing from the voxel selection to the sorted matrices.

preserve the first-order statistics of the stimuli while removing any semantic content-even though we cannot completely exclude some preservation of low-level correlations. The temporal sequence of the frames was kept the same as in the original movie. First, all cinematic cuts were marked. Next, each shot (i.e., the continuous video sequence between two cuts) underwent a spatiotemporal Fourier phase scrambling procedure (Froudarakis et al., 2014): The shot video was represented as a 3D matrix (height $\times$ width $\times$ time), which was Fourier transformed, element-wise multiplied with a random phase matrix, and then transformed back into time domain. For a given shot, this was repeated for the three color components, using the same random phase matrix. The usage of the same random phase matrix ensured that all of the colors found in the scrambled shots were mixtures of colors that appeared in the unscrambled shots. The resulting video preserved the spatiotemporal power spectrum of each shot, its color range, and the flicker induced by cinematic cuts between shots, without including any discernible visual objects (see video 1 ). The entire audio track underwent a time domain multiband audioscrambling procedure (adapted from (Ellis 2010; MinagawaKawai et al., 2010)) and slightly modified to fit our particular audio track characteristics. The audio signal was passed through a 256 channels' gamma tone filter bank. Then, it was divided into $25 \mathrm{~ms}$ long partially (50\%) overlapping Hanning windows. Within each sub-band independently, the windows were randomly displaced according to a Gaussian jitter of a $500 \mathrm{~ms}$ of standard deviation. As in the color components of the video, both stereo channels were shuffled in a congruent manner. This procedure resulted in an audio track that followed the local spectrum and amplitude envelope of the original soundtrack in a scale of a second while rendering speech indiscernible (video 1).

For both movie and scrambled-movie sessions, participants were instructed to focus on the movie and avoid mind wandering.

\section{Categories 1-Back Task}

All participants took part in the categories 1-back experiment. Visual stimuli consisted of colored pictures of faces (Minear and Park 2004), houses, body parts (free-copyrights Google images), tools (BOSS database (Brodeur et al., 2010)), and textures, including a fixation point. All pictures $(10 \times 10 \mathrm{deg})$ were presented for $200 \mathrm{~ms}$ on a gray background. The experiment comprised two 9-min long scans, in which stimuli were shown in 10-s blocks and alternated with $6 \mathrm{~s}$ of a gray background. Subjects were instructed to push a button with their right hand every time two identical items appeared in a row.

\section{Shape Recognition Task}

A total of 12 participants out of 15 took part in the shape recognition task. The presently analyzed condition was acquired during an experiment primarily designed to measure the activity modulation in object areas and is fully stated by Goldberg et al. (2016). Stimuli consisted of shapes composed of 10 contiguous identical light or dark green squares, created in a shapesearch computer game, by either 101 human players or by a random walk algorithm (Noy et al. 2012).

The experiment comprised four types of blocks, with each block composed of nine homogeneous-category shapes (one second each): light green shapes created by humans, light green shapes created by the algorithm, dark green shapes created by humans, and dark green shapes created by the algorithm. During the fMRI scan, each block was $9 \mathrm{~s}$ long, followed by a 9-s fixation period. 29 blocks were presented in total. Subjects were instructed to look at a fixation point and perform a task related to the features of the stimulus (further description of the experiment appears in Goldberg et al., 2016).

\section{Experimental Setup and Imaging Parameters}

The fMRI experiments were conducted at the Weizmann Institute of Science (Rehovot, Israel) using a $3 \mathrm{~T}$ Tim Trio scanner (Siemens Medical Systems, Erlangen, Germany). Single-shot echo-planar imaging (EPI) images were acquired with interleaved slice ordering using a standard receive-only 12-channel head matrix coil. Each participant underwent 3-4 fMRI scanning sessions in separate days. The order of the scanning 
sessions was counterbalanced across subjects, with an exception that the sessions always began with one resting-state run.

For the shape recognition and categories 1-back tasks, 46 slices ( $3 \mathrm{~mm}$ thick, no gap, interleaved excitation order, inplane resolution $3 \times 3 \mathrm{~mm}$ ) parallel to the anterior-posterior commissural plane were collected. Each run included 178 for the shape recognition task and 240 for the object recognition task single-shot EPI images per slice (repetition time [TR], $3000 \mathrm{~ms}$; echo time [TE] $30 \mathrm{~ms}$, flip angle $75^{\circ}, 80 \times 80$ matrix for the shape recognition task and $70 \times 70$ matrix for the shape recognition task; bandwidth $2404 \mathrm{~Hz}$ /pixel; FOV $240 \mathrm{~mm}$ ).

For the rest, movie, and scrambled-movie conditions, 34 slices $(3.3 \mathrm{~mm}$ thick, no gap, interleaved excitation order, inplane resolution $3 \times 3 \mathrm{~mm}$ ) parallel to the anterior-posterior commissural plane were collected. Each run included 240 for rest, 270 for the movie and scrambled-movie single-shot EPI images per slice (TR, $2000 \mathrm{~ms}$; TE $27 \mathrm{~ms}$, flip angle $75^{\circ}, 70 \times 70$ matrix; bandwidth $2380 \mathrm{~Hz} /$ pixel; FOV $240 \mathrm{~mm}$ ).

Overall, 6 fMRI runs were carried out in each of the 15 subjects (2 runs of resting-state EC, 2 runs of movie, and 2 runs of visual categories 1-back task; see Fig. 1A). Moreover, 4 additional fMRI scans were carried out in 12 out of 15 subjects (shape recognition task and rest EO), and 2 fMRI scans were carried out in 14 out of 15 subjects (scramble movie), for a total of 166 fMRI runs.

Two structural scans were carried out in each participant (T1-weighted sagittal Magnetization Prepared Rapid Gradient Echo (MPRAGE) sequence, 176 slices, $\mathrm{TI}=900 \mathrm{~ms}$, TE $=2.98 \mathrm{~ms}$, $\mathrm{TR}=2300 \mathrm{~ms}$, flip angle $=9^{\circ}, 256 \times 256 \times 176$ matrix, $1 \mathrm{~mm}^{3}$ voxels, bandwidth $=240 \mathrm{~Hz} /$ pixel).

\section{Data Analysis}

\section{fMRI Data Preprocessing}

All fMRI data were processed using FSL 5.0.2.1 (www.fmrib.ox. ac.uk/fsl) and in-house Matlab code (MathWorks). Functional data were analyzed using FMRIB's expert analysis tool (FEAT, version 6). The following prestatistics processing was applied to the data of each participant: motion correction using FMRIB's Linear Image Registration Tool (MCFLIRT) (Jenkinson et al., 2002); brain extraction using BET (Smith et al., 2009); and high-pass temporal filtering with a cutoff frequency of $0.01 \mathrm{~Hz}$. Functional images were aligned with high-resolution anatomical volumes initially using linear registration (FLIRT), then optimized using boundary-based registration (Greve and Fischl 2009). Structural images were then transformed into standard MNI space using nonlinear registration tool (FNIRT), and the resulting warp parameters were applied to the functional images as well. All the functional images were resampled to $2 \times$ $2 \times 2 \mathrm{~mm}^{3}$ standard space. We defined the motioncontaminated frames using the framewise displacement (FD) and the DVARS measure (temporal derivative of root-meansquare blood oxygen level-dependent (BOLD) signal of all within-brain voxels) (Power et al., 2012, 2014; Siegel et al., 2016; Ciric et al., 2017). The FD threshold was set to $0.3 \mathrm{~mm}$ and DVARS censoring threshold was set at $0.5 \%$ root-mean-square frame-to-frame intensity change. The thresholds were chosen in order to have a minimum of $\sim 5 \mathrm{~min}$ of data per run (Power et al., 2014). For each frame contaminated by motion, we additionally censored one preceding and one following frame, due to uncertainty in the precise timing of the movement (Siegel et al., 2016). The fraction of uncensored frames was as follows: 99\% for rest EC, $91 \%$ for rest EO; $97 \%$ for movie and categories 1 back, $96 \%$ for scrambled, and $90 \%$ for shape recognition.
Moreover, for each subject, run, and experimental condition, we extracted the average motion amplitude (absolute displacement values in $\mathrm{mm}$, MCFLIRT output). The two runs within each condition were averaged to generate a single mean motion value per condition. We then ran a one-way mixed ANOVA to compare the mean motion values between different conditions.

Tissue-type segmentation was carried out using FAST (Zhang et al., 2001), and ventricles and white matter masks were drawn. The non-neuronal contributions to the BOLD signal were removed by linear regression of motion parameters, ventricle and white matter timecourses for each participant (Fox et al., 2009). The white matter and ventricles of each participant were automatically defined using FSL's FAST (Zhang et al., 2001) and refined to avoid boundaries between tissues (Hahamy et al., 2015).

An additional nuisance regressor was derived from the global signal averaged over the brain (Fox et al., 2009; Power et al., 2014). The correlation analysis was repeated without regressing out the global signal and yielded similar results (see Supplementary Figure 2). No spatial smoothing was performed, to maintain the maximal amount of detailed information in the data.

\section{Voxelwise Functional Connectivity Maps}

A single-subject standard functional connectivity analysis was applied to the resting-state scans for a demonstrative purpose: Single voxels in the category-selective areas were each taken in turn as a seed (one-voxel seed), and Pearson's correlation between each seed voxel's timecourse and all other cortical voxels' timecourses was calculated in Matlab. A functional connectivity map based on a single voxel as a seed was obtained for each of the two resting-state runs. The maps of the first and second resting states were then averaged to reduce noise in the resting-state correlation pattern estimates. Average MNI maps were registered to a common FreeSurfer template surface hemisphere (fsaverage), for display purposes only. For the visually driven conditions (movie and scrambled-movie viewing, shape recognition, and categories 1-back task), we replaced the standard within-run functional connectivity analysis with a between-run functional connectivity analysis to isolate stimulus-driven activity, while avoiding intrinsic, nonstimulusrelated effects (Golland et al., 2007; Hasson et al., 2010). Therefore, functional connectivity was calculated only between the two runs of each participant in the following manner: Each seed voxel's timecourse was taken from the first movie presentation and was correlated with all the voxel timecourses of the second movie presentation. The same procedure was then applied to the second versus first movie presentation, that is, each seed voxel's timecourse was taken now from the second movie presentation and was correlated with all the voxel timecourses of the first movie presentation. Thus for each seed voxel, two maps were created, which were then averaged and projected onto FreeSurfer cortical surface template using the same procedure described above. This procedure resulted in multiple maps with an $r$ value for each voxel, from which one example map with a seed voxel sampled in the extrastriate body area (EBA) is represented in Figure 2.

To better visualize the connectivity pattern regardless the overall correlation strength (which may differ between participants), the correlation values in each matrix were normalized by removing the mean and by dividing each correlation value by the 99.5th percentile of the absolute values of each square 


\section{A Visual conditions}

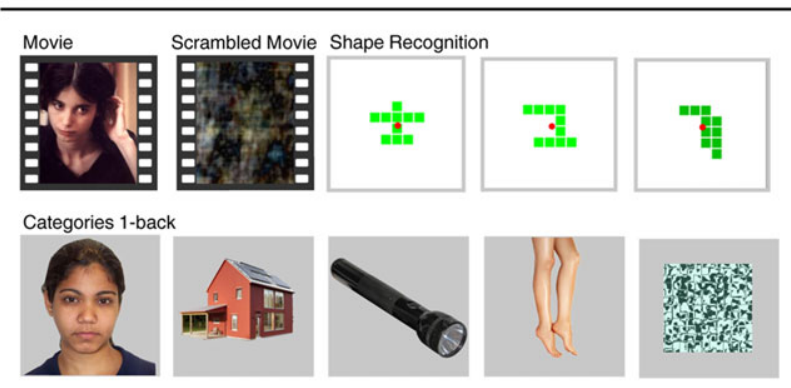

B Region of Interest

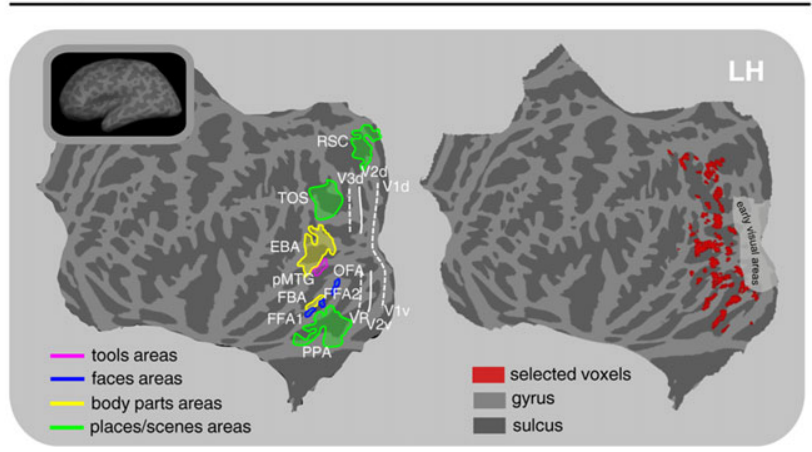

Figure 2. Experimental conditions and definition of region of interest. (A) The panel shows sample images of the visual-evoked activity conditions. Top row: a frame extracted from the movie Broken Wings (courtesy of Nir Bergman), a frame from the scrambled movie, and some sample images used in the shape recognition task. Bottom row: some sample images used in the categories 1-back task. Each condition had two runs, and the order of conditions was counterbalanced across participants with the exception that each session always began with a resting-state run. (B) The panel shows a flattened representation of the left hemisphere for a representative participant. In the right panel, the hemisphere is overlaid with the region of interest as defined by all of the visual conditions. Early visual areas were excluded from the analysis (see Methods). As a comparison, in the left panel, the hemisphere is overlaid with category-selective areas as defined by the categories 1-back condition using a standard activation-preference approach. Note that these areas were not used in the analysis and are shown here only as a reference.

matrix (thus reducing the effect of the extreme $1 \%$ outlier values on the matrix color range).

\section{Selection of Voxels for Pattern Extraction}

To identify voxels located in higher order visual areas, we adopted the following voxel selection process. For each participant and each voxel, the timecourses of BOLD responses during all visual conditions (movie, scrambled movie, categories 1back, shape recognition) were sequentially concatenated into a single long timecourse. This was done separately for the first and second runs, resulting in two long timecourses. Note that the sequence of stimuli in the two runs were identical. We then calculated the cross-run correlations between the timecourses for the first and second runs and identified voxels with correlations above 0.25 between the two runs (Golland et al., 2007; Hasson et al., 2010) (Fig. 1 gives a visual description of the analysis). This threshold was chosen since it represents a good trade-off between the magnitude of the correlation coefficient and a large enough sample of selected voxels per subject (the minimum number of selected voxels was 883 ). The same analysis was then repeated for thresholds of $0.20,0.30$, and 0.4 and yielded comparable results (see Supplementary Figure 1). From these sets of voxels, we selected only those located in higher order visual areas. This procedure was performed for each subject individually, creating an individual cortical mask based on the Harvard-Oxford cortical atlas (as implemented in FSL; Makris et al., 2006). For the main analysis, early visual areas and nonvisual areas were removed from the selection. The same analysis was then repeated excluding only the early visual areas while retaining responsive voxels in nonvisual areas across the entire cortex.

It should be noted that all visual conditions contributed to the selection process and all had roughly equal acquisition duration (see "Contribution of the Visual Conditions to the Model Selection" for more details). For nine subjects, the location of voxels removed according to the atlas was then compared with the location of early visual ROIs defined according to standard retinotopic mapping (Sereno et al., 1995; Wilf et al., 2015) and found appropriately located (even though we cannot exclude that a few voxels belonging to the peripheral portions of the early visual areas may have been included in the analyses). Figure $1 \mathrm{~B}$ shows an example of selected voxels defined in the left hemisphere of a representative subject (note that the analysis was performed in both hemispheres). Just for comparison, we defined in this representative subject categoryselective areas based on standard criteria using an independent set of scans (two supplemental runs of categories 1-back task). The first (premagnetization steady state) four volumes were discarded. Motion correction and cross-scan alignment were performed using the Analysis of Functional NeuroImages (AFNI) $3 \mathrm{dvolreg}$. The preprocessed data were averaged and registered to MNI space. After the composition of transforms, the functional data were resampled in one step to $3 \mathrm{~mm}$ isotropic voxels. We used a general linear model (GLM) with blocks modeled as boxcar functions and convolved with a canonical hemodynamic response function. For each category, we computed a statistical parametric map based on a contrast between two conditions. All maps were thresholded at $P<0.001$ (not corrected) and projected on the participant's cortical surface with $3 \mathrm{~mm}$ FWHM (full width at half maximum) of surface smoothing and cluster correction for multiple comparisons at 0.01 (Hagler et al., 2006). Face-selective areas (FFA1, FFA2, and OFA) were defined based on the contrast faces > scenes (Kanwisher et al., 1997). Sceneselective areas (PPA, RSC, and TOS) were defined based on the contrast scenes $>$ faces (Epstein and Kanwisher 1998). Body parts-selective areas (EBA and FBA) were defined based on the contrast body parts > objects/tools (Downing et al., 2001). Toolsselective area (pMTG) was defined based on the contrast objects/ tools $>$ body parts (Lewis 2006). All the analyses were performed with FreeSurfer (Dale et al., 1999; Fischl et al., 2002). A singlesubject example of such standard division to category-selective areas is depicted in Figure 2B.

\section{Voxelwise Correlation Matrices}

To obtain the full pairwise correlation matrix of all selected high-order visual voxels (mean \pm SD $1686 \pm 516$ voxels per participant), we extracted the timecourses of all selected voxels under each experimental condition. A pairwise Pearson's correlation matrix was then calculated for all voxels. For all the visual conditions, the correlation matrices were calculated between runs. Briefly, the timecourses of all the voxels in the first run were correlated with the timecourses of all the voxels in the second run and vice versa. This procedure formed an asymmetrical matrix with the diagonal representing the timecourse correlation of the same voxel across the two stimuli presentations (similar to Golland et al., 2007). Values below the 
diagonal represented correlations of each of the seed voxels taken from the first run with all the voxels taken from the second run (and vice versa for values above the diagonal).

For resting-state runs, the correlation matrices were calculated within each run, and an average of the two within-run matrices was calculated to emphasize consistent correlation patterns and to reduce noise. This procedure formed a symmetrical matrix.

\section{Volumetric Distance Analysis}

The specific pattern of spontaneous correlations could be the result of their anatomical vicinity (i.e., adjacent voxels would show high correlations, while distant voxels would be less correlated). To check for this possible confound, we calculated a volume distance model. The volumetric Euclidean distance was calculated in 3D anatomical space for each pair of voxels, and all distances were inverted (1/distance) so that closer voxels had higher values and distant voxels had lower values.

We used Spearman's coefficient to assess the effect of the volumetric distance between voxels on the correlation patterns because this coefficient can be used to compare variables on different scales that are not necessarily linearly related (a vector of correlations and a vector of distances).

\section{Sorted Matrices}

To better visualize the level of resemblance between the resting-state and stimulus-driven connectivity patterns, we rearranged the voxels in all the matrices according to the resting-state correlation matrix.

First, the selected voxels in the within-run resting-state matrices were sorted based on the correlation strength according to a descending criterion (from the strongest to the weakest correlation). Since these matrices are symmetrical, only values below the diagonal were taken. Then, these sorted values were rearranged within a shape of a square, showing a salient pattern of correlation gradually moving from the strongest to weakest values along a winding diagonal path (see Fig. $1 \mathrm{C}$ in Wilf et al. (2015) for demonstration). It is important to note that the location index of each correlation in the original restingstate matrix was kept so that the same index order could be applied to the stimulus-induced matrices as well.

Because the original cross-run stimulus-evoked matrices were not symmetrical, both values below and above the diagonal had to be taken into account. This was done by averaging each value below the diagonal with its homologous value above the diagonal (i.e., the correlation of voxel $i$ from the first run with voxel $j$ from the second run was averaged with the correlation of voxel $j$ from the first run with voxel $i$ from the second run). Then, both the stimulus-evoked and distance matrices of each participant were rearranged according to the resting-state matrix correlation strength. This sorting procedure ensured that if the original correlation pattern of a certain condition was similar to the resting state, the new sorted square would present a gradual diagonal pattern similar to that of the corresponding resting-state square.

In order to average the sorted squares across participants, we matched the square size. Because different participants had different original matrix sizes, the size of the mean square was determined by the subject with the smallest number of selected voxels in the square. Therefore, only a subset of the sorted correlation values was taken, to form for each participant a $361 \times 361$ size square for the region of interest. The values were sampled in constant intervals across the sorted vector forming the square so as to cover the whole range of correlation values of each subject. These values were then averaged across subjects, turned back into a square matrix, and used for visualization of the comparison between different conditions.

\section{Correlations Between Spontaneous and Stimulus-Induced Connectivity Patterns}

For each participant, we examined the similarity between the resting-state and each of the stimulus-driven connectivity patterns. To that end, each unsorted matrix was transformed into a vector in the following manner: For the asymmetrical stimulus-driven matrices, the values below and above the diagonal were transformed into vectors and then averaged (such that values were averaged symmetrically). For the averaged resting-state matrices, only values below the diagonal were transformed into a vector, as the matrices were symmetric. The values along the main diagonal were not taken into account in the comparison because the resting-state diagonal is uninformative (by definition all the correlations values in the diagonal are equal to one). The symmetrical distances matrices were also turned into a vector like the resting-state matrices. Then Pearson's correlation between the resting-state vector and each of the stimulus-driven vectors was calculated for each participant. For comparison between resting-state vector and the volumetric distance vector, a nonparametric Spearman correlation was used (the correlation was done after the distances were inverted to 1 /distance). All statistical tests were performed at the second level (group) after correlation values underwent Fisher's $r$ to $z$ transformation. Hence, we did not rely on the various auxiliary assumptions involved with testing correlation coefficients, such as independency and normality. One-sample t-tests were used to establish whether the correlations between the matrices of each condition and the resting-state matrices were significantly greater than zero, and $P$-values were Bonferroni corrected (taking into account the number of conditions).

Then, to assess the similarity between the stimulus-induced patterns and the resting-state pattern, a mixed-effect one-way repeated-measures analysis of variance (ANOVA) (Bates et al., 2014) was performed. The experimental condition served as the fixed-effects factor and subjects provided random intercept. Correlation values were Fisher-transformed before the mixedeffects model was fitted. The main effect of condition was tested using Kenward-Roger approximation for degrees of freedom as implemented by R package "afex" (Singmann et al., 2015). Least squares means and all pairwise condition simpleeffects were estimated by R's "lsmeans" command. All post hoc t-tests were false discovery rate (FDR) corrected.

\section{Reliability Measurement}

To assess if the stimulus-driven response elicited by each visual experiments was consistent across runs, we estimated the reliability of the correlation patterns. It is important to note that for each pair of voxels and each visual experiment, we calculated two independent correlation measures: The first measure was obtained by correlating the first voxel timecourse during the first stimulus presentation with the second voxel timecourse during the second stimulus presentation; the second measure was obtained by correlating the first voxel timecourse during the second stimulus presentation with the second voxel timecourse during the first stimulus presentation. By comparing these two values, we could get a measure of the reliability of the overall correlation set. Thus, the vector of 
values below the diagonal was correlated with the vector of values above the diagonal to give the reliability measure for each matrix. A mixed-effect one-way repeated-measures ANOVA was then performed on the single-subject correlations taking the experimental conditions as a factor, followed by FDRcorrected post hoc t-tests (see Correlations Between Spontaneous and Stimulus-Induced Connectivity Patterns for details).

\section{Comparison Between Resting State Before and After the Movie Presentation}

In this analysis, resting-state runs were not averaged. After creating the functional connectivity matrices and turning them into vectors (as described above), each vector was independently correlated with the cross-movie vector.

\section{Comparison Between the First and the Second halves of Each Task Run}

For each subject and experimental condition, we split each run into two segments of equal length (the first half of the volumes and the second half of the volumes). We then performed our main analysis while taking into account once the first half of each run, and once the second half of each run. In other words, the first or the second half of the visual conditions was correlated with either the first or the second half of the first restingstate run (Geerligs et al., 2015; Vanderwal et al., 2017). We then compared the results of the first half with the results of the second half by performing a mixed-effect two-way repeatedmeasures ANOVA. The analysis was performed on the withinsubject correlations with the factors visual condition (five levels: movie, scrambled movie, category 1-back task, shape recognition task, and rest) and segment (two levels: first half and second half), followed by FDR-corrected post hoc t-tests.

\section{Comparison Between Rest EC and Rest EO}

For this control experiment, we repeated the same analyses performed for rest with EC (see Fig. 6A,B). In addition, we directly assessed the difference between rest with EC and EO by performing paired t-tests across the visual conditions (Fig. $6 \mathrm{C}$ ).

\section{Contribution of the Visual Conditions to the Model Selection}

To assess the contribution of each visual condition to the voxel selection, and to rule out the possibility that the movie condition primarily drives the correlations across the concatenated runs, we compared the cross-run correlation within each visual condition with the cross-run correlation calculated concatenating all the visual conditions. In particular, for each subject and visual condition, we generated a voxel-by-voxel cross-run map (each voxel was correlated with itself between the two-run repetitions; Golland et al., 2007). Then, each visual condition map was correlated to the voxel-by-voxel cross-run map generated by concatenating all the visual conditions. Finally, we performed a one-way mixed ANOVA to assess the similarity between the concatenated map and each visual condition map.

\section{Searchlight Task-Rest Correlation Analysis}

To inspect the regional variations of the correlated tasks and rest activation patterns across the entire cortex, we repeated the analysis described above with a searchlight approach (Kriegeskorte et al., 2006). For each given task (e.g., natural movie viewing), a searchlight correlation map was calculated for each participant. These maps reflect the local correspondence between resting-state functional connectivity, averaged across the two rest scans, and task-related functional connectivity, estimated by correlating between a pair of task scan repetitions.

First, for each individual, a cortical mask was constructed by intersecting an anatomical gray-matter mask (FSL segmentation) and a brain mask, which consisted of only voxels showing above-zero temporal BOLD variation in the two task scans and the two rest scans. Then, we built a spherical ROI (region of interest) around each voxel included in the cortical mask, with a radius of three voxels. Voxels outside the cortical mask were removed from the spherical ROIs, so effectively their shape was not necessarily spherical but followed the contours of the individual subject's cortex. To ensure reliable correlation estimates, spherical ROIs left with $<30$ voxels were discarded.

Within each spherical ROI, we linearly correlated the time series of each voxel with the time series of each other voxel. In the rest scans, this was done within each of the two scans. In the task scans, the correlation was calculated across the two scans. The same analysis, as described in the "Correlations Between Spontaneous and Stimulus-induced Connectivity Patterns," was performed for each of these spherical ROIs. Briefly, for a spherical ROI of 100 voxels, this stage resulted in three $100 \times 100$ correlation matrices. The two correlation matrices resulting from correlating within the rest scans were averaged together and their lower (or equivalently, upper) off-diagonal elements were extracted as a rest-related spatial pattern vector (e.g., for a 100 voxels sphere, the vector was 4950 voxels long). The correlation matrix resulting from correlating the two task scans was averaged with its transpose and then the lower (or equivalently, the upper) off-diagonal elements were extracted to form a taskrelated spatial pattern vector.

We then conducted a second Pearson correlation between the rest-related and task-related spatial pattern vectors corresponding to each spherical ROI. This correlation coefficient was assigned to the central voxel of the ROI. This procedure formed a full cortical map of local rest task regional BOLD correspondence for each participant and each task. For each task, these maps were Fisher-transformed and averaged across participants and then registered to the fsaverage surface with FreeSurfer.

\section{Results}

In the current study, we examined the relationship between spontaneous activity in higher order visual areas and the response to naturalistic visual stimuli. To that end, we compared the connectivity patterns emerging spontaneously in higher order visual areas during rest with those produced during naturalistic movie viewing and in response to more artificial stimuli that isolated specific aspects of the movie stimulus (motion, naturalistic images, or appearance of objects). Participants underwent fMRI scans that included six different conditions, each repeated twice (Methods and Fig. 2A). The experiment consisted of two 8-min resting-state runs with EC (15 participants), two 8min resting-state runs with EO and fixation (9 participants), and two 8-min runs of each of the following visual conditions: naturalistic movie viewing (engaging movie segment; 15 participants), scrambled movie viewing (phase-scrambled in the space domain; 14 participants), a categories 1-back task (block-design paradigm with five different object categories; 15 participants), and a shape recognition task (block-design paradigm with light and dark green artificial shapes; 12 participants).

As a preliminary analysis, we calculated for each condition the average motion amplitude (Mean \pm SD: rest $0.22 \pm 0.09$; movie $0.27 \pm 0.21$; categories 1-back task $0.22 \pm 0.10$; scrambled movie $0.26 \pm 0.015$; shape recognition task $0.14 \pm 0.05$; rest EO 


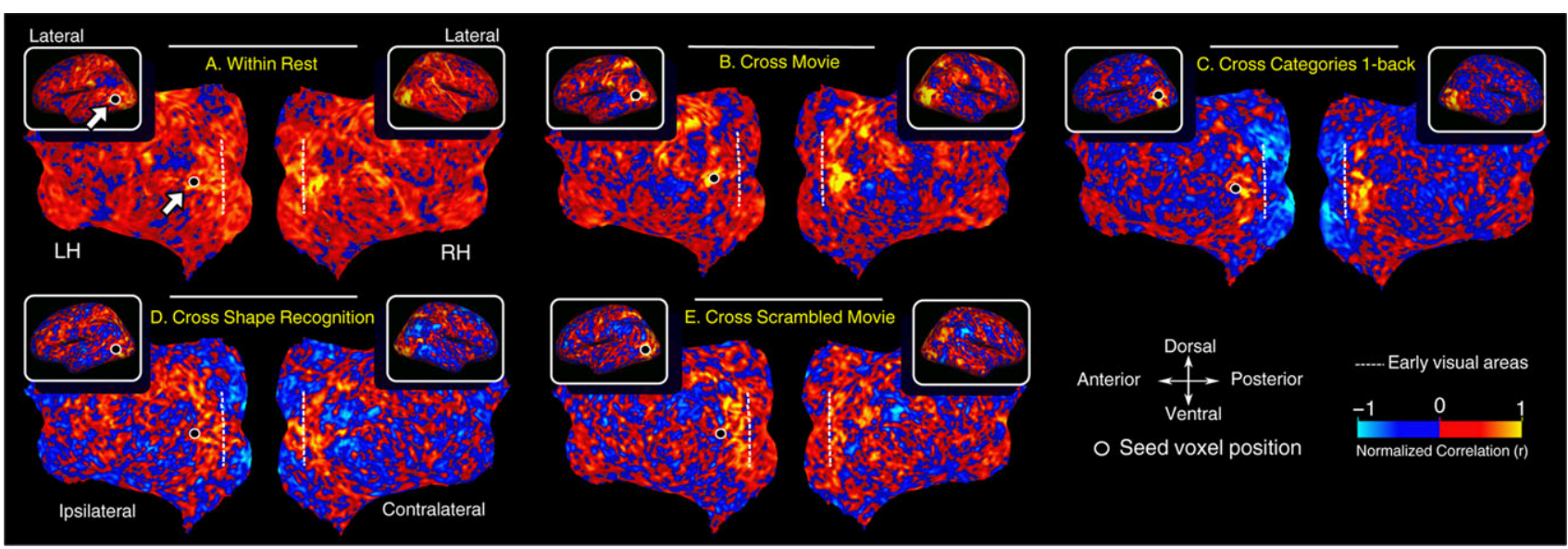

Figure 3. Similarity between resting-state and movie voxelwise functional connectivity maps in a representative participant. (A) The left and right hemispheres of a representative participant are overlaid with a within-resting-state functional connectivity map with a seed voxel in the left EBA, marked with a black dot. (B) Connectivity map across movie presentations using the same seed voxel. Note that the same general pattern arising spontaneously in the resting-state map is also present in this movie-driven map. (C) Connectivity between categories 1-back scans (cross-run) using the same seed voxel. Note some similarity between the positive correlations in this map and the resting-state correlations and anti-correlations (in blue) in the early visual areas. (D) Cross-run connectivity map for the shape recognition task and $(E)$ scrambled movie. Maps were overlaid on the Freesurfer fsaverage flat cortical surface (see Methods). Correlation values $r$ were normalized (for display purposes only)

$0.28 \pm 0.17)$. The one-way mixed ANOVA yielded a significant difference in the average motion amplitudes across conditions $(\mathrm{F} 5,62.76=3.53, P=0.007)$. In particular, we found a significantly lower average motion amplitude in the shape recognition task compared with movie $\left(t_{63}=3.39, P=0.009\right)$, scrambled movie $\left(t_{64}\right.$ $=3.23, P=0.009)$, and rest $\left(t_{64}=3.68, P=0.007\right)$. There was no statistically significant difference between the other conditions.

In our main analysis, we focused on higher order visual areas, identified as voxels responding to all visual conditions (Methods, "Selection of voxels for pattern extraction"). We aimed to include the full set of category-selective higher order visual voxels activated during the tasks rather than limiting the analysis to specific categorical preferences.

Figure $2 B$ illustrates the layout of these areas in a representative participant: The right panel shows an example of the voxels selected for further analysis, while the left panel shows category-selective areas defined using standard criteria, as a comparison. Note that all voxels sampled were located in the visual cortex.

\section{Resting-State Patterns Most Strongly Resemble Naturalistic Stimulus-Induced Patterns}

Examining seed-based connectivity patterns emerging spontaneously during the resting state and patterns evoked by naturalistic and artificial visual stimuli revealed a widely distributed pattern. Figure 3 depicts an example of one such pattern. The maps show the correlation values for the timecourse of each cortical voxel with the timecourse of a seed voxel (black dot) located in the EBA of a representative participant. Importantly, while resting-state correlations were calculated using timecourses extracted from the same run (within-run correlations) and then averaged across the two resting-state conditions, correlations for the visual conditions were calculated across two runs (cross-run correlations).

In agreement with previous reports (Hutchison et al., 2014), the correlation patterns that emerged during rest with EC (Fig. 3A) showed a bilateral, symmetrical, widespread pattern in the superior temporal gyrus, middle temporal gyrus, insula, and early visual cortex. We compared the resting-state pattern with the patterns induced by the same seed voxel across the two repetitions of each visual condition (Fig. 3B-E). The maps show that free movie viewing produced the functional connectivity pattern that most strongly resembled the widely distributed resting-state pattern. In contrast, both the categories 1-back and the shape recognition task produced a more localized pattern, mainly around EBA, with some negative correlations in the peripheral ventral areas of the striate cortex. Finally, the scrambled movie seemed to produce a distributed, although lessorganized, correlation pattern, likely due to a noisier response to this type of stimulus.

To allow for visual inspection of the entire set of voxelwise connectivity patterns across the various experimental conditions, we adopted the same method that we used previously for representing the connectivity structure in early visual areas (Wilf et al., 2015). Briefly, for each condition, we calculated a matrix of all pairwise correlations of higher order visual voxels in both hemispheres (Methods, "Voxelwise Correlation Matrices"). To better visualize potential similarities between patterns across conditions, cells were rearranged to match the resting-state matrix (Methods, "Sorted Matrices"). Note that this artificial rearrangement of the matrices was for visualization purposes only and did not affect the subsequent statistical analyses.

As a comparison, Figure $4 \mathrm{~A}$ shows the unsorted correlation matrices in a representative subject during rest, cross-movie, categories cross-1-back, cross-scrambled movie, cross-shape recognition task, cross-rest, and Euclidean distance.

Figure $4 B$ shows the results of this sorting procedure. Similarities between the resting state and other conditions are reflected as comparable color gradients, allowing for a visual comparison between the cross-run correlation patterns for each visual condition and the within-run correlation pattern for the resting state (each pattern represented by an individual square). Figure $4 B-2$ shows the rearranged mean matrix of pairwise cross-run correlations driven by naturalistic movie viewing. Although some degradation in the similarity to the restingstate pattern can be discerned, the re-emergence of the 
underlying structure is evident. The other panels show the rearranged matrices of pairwise cross-run correlations for the categories 1-back task, the shape recognition task, scrambled-movie viewing, and the cross-resting-state runs. All visual conditions showed some trace of the resting-state pattern; however, the resting-state pattern most closely resembled the movie-driven correlation matrix. The cross-resting-state matrix showed a noisy pattern with correlations near zero, as expected given the inherent inconsistency between rest runs.

We subsequently quantified the similarity between restingstate and stimulus-driven patterns (Methods, "Correlation Between Spontaneous Connectivity and Stimulus-Driven Patterns"), shown in Figure 4C. The y-axis represents the correlation between the cross-run correlations for each visual condition and the within-run correlations during rest. In other words, the bars represent the similarity between the pairwise correlation pattern for each visual condition and the pairwise correlation pattern during rest. All visual conditions showed a significant correlation with the resting-state pattern (smallest $\left.t_{11}=3.09, P=0.010\right)$. However, correlations between the resting and movie-viewing conditions were significantly higher than between the resting and artificial visual stimulus conditions (movie vs. categories 1 -back, $t_{52}=2.83, P=0.008$; movie vs. scrambled movie, $t_{52}=3.33, P=0.002$; movie vs. shape recognition, $t_{53}=6.19, P<0.0001$; post hoc $t$-test, FDR corrected). As a control, we computed a between resting-state scan correlation matrix, assuming no consistent pattern should emerge (Panel B6). Indeed, this cross-rest condition showed no significant similarity to the within-resting-state pattern, as expected given the spontaneous nature of fluctuations during resting-state runs.

We next repeated the same analysis excluding only the early visual areas while retaining the nonvisual responsive voxels across the entire cortex. Overall, the pattern of results was similar to the one obtained in the higher order visual areas (Supplementary Fig. 3). All visual conditions showed a significant correlation with the resting-state pattern (smallest $t_{12}=35.72$,
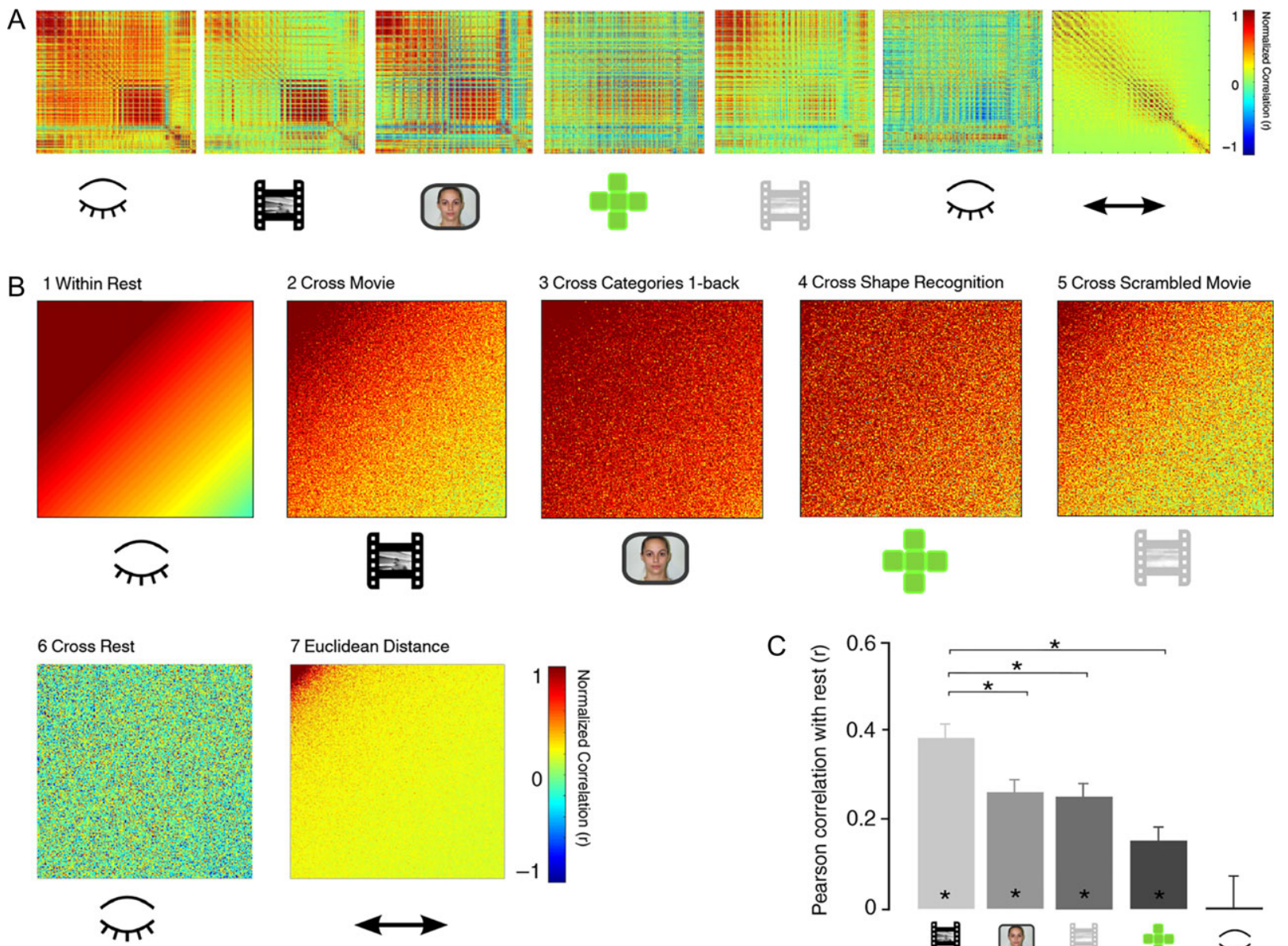

Figure 4. The resting-state pattern is more strongly correlated with naturalistic movie-induced patterns than with patterns evoked by more artificial visual conditions. Panels A and B show the unsorted and sorted matrices of all pairwise correlations of higher order visual voxels (corresponding to the region of interest) in both hemispheres, for the resting state, each of the visual conditions, and volumetric distance. (A) Unsorted mean correlation matrices in a representative subject during rest, movie, categories 1-back, scrambled movie, shape recognition task, cross-rest, and Euclidean distance. (B) 1 . The resting-state pairwise correlation matrix of all voxels in the region of interest was sorted according to correlation strength to form a diagonal pattern from highest to lowest correlation (see Methods). The mean matrix across all participants is presented $(n=15)$. 2. The voxel order from the resting-state matrix was applied to the cross-movie matrix and averaged across participants. Note that the diagonal pattern reemerges in the naturalistic movie condition $(n=15)$. $3-5$ Same as 2 , 3. but for categories 1 -back $(n=15)$, 4. shape recognition $(n$ $=12)$, and 5. scrambled-movie $(n=14)$ conditions. Note that some similarity to the resting-state pattern remains, but the similarities are much less salient than for the naturalistic movie. 6. Cross-resting-state pattern; 7. Euclidean distance. (C) The bars show the mean correlation between the within-run resting-state pattern and the cross-run visual patterns. All visual conditions showed a significant correlation with the resting state, but the movie showed a significantly higher correlation as compared to the other visual conditions. The cross-resting-state matrix did not show a significant correlation with the within-run resting-state matrix (rightmost bar). Error bars denote the standard error of the mean $\left.( \pm \mathrm{SEM}){ }^{*} \mathrm{p}_{\mathrm{FDR}}<0.05\right)$. 
$P=0.0001)$. However, correlations between the resting and movie-viewing conditions were significantly higher than between the resting and shape recognition task and scrambled movie (movie vs. categories 1-back, $t_{52}=2, P=0.05$; movie vs. scrambled movie, $t_{52}=2.67, P=0.01$; movie vs. shape recognition, $t_{53}=4.62, P<0.0001$; post hoc $t$-test, FDR corrected).

\section{Similarities Between Resting-State and Naturalistic Stimulus-Induced Patterns are not Driven by Cross-Run Reliability}

An important factor that could explain the preferential correlations between resting-state and movie-driven patterns is the reliability of responses (Golland et al., 2007). In other words, the movie could be more engaging, thus producing a stronger and more reliable BOLD response than the more artificial conditions. It is important to emphasize that the stimulus-evoked patterns were all based on cross-run correlations. Therefore, a stimulation condition leading to unreliable responses would inevitably result in a noisier correlation pattern that would not be expected to resemble the resting-state pattern. To address this issue, we measured response reliability by calculating the reproducibility of the connectivity patterns. A simple measure of this reproducibility is provided by the mirror symmetry across the diagonal of each unsorted cross-run correlation matrix (Methods, "Reliability Measurement").

In Figure 5, the $y$-axis represents the reliability measures for the different visual conditions. As expected, all stimulus-driven tasks showed a significantly reliable pattern (smallest $t_{14}=5.12$, $P<0.0001)$. No significant reliability was found across restingstate runs when the cross-run correlations were assessed, which is again consistent with the spontaneous nature of these fluctuations. Importantly, despite its stronger similarity to resting-state patterns, the reliability of the movie-driven pattern was significantly lower than that of the categories 1-backdriven pattern $\left(t_{52}=6.45 ; P_{\mathrm{FDR}}<0.0001\right.$; post hoc $t$-test movie vs. categories 1-back, FDR corrected).

\section{Similarity Between Resting-State and Naturalistic Stimulus-Induced Patterns cannot be Explained by Volumetric Distance}

The specific pattern of spontaneous correlations could be the result of the volumetric distance between voxels (i.e., anatomically-adjacent voxels would show higher correlations, while far-away voxels would be less correlated). To assess the effect of the anatomical distance between voxels on the correlation patterns, we measured the inverted Euclidean distance between each pair of voxels and assigned it to the sorted restingstate matrix (Methods, "Volumetric Distance Analysis"). As expected, there was some contribution of anatomical distance to the resting-state correlation pattern, but this effect alone could not account for the spontaneously emerging patterns. Thus, the correlations between the resting state and movie-viewing task were higher than between the resting state and the anatomical distance between voxels (movie vs. volumetric distance, $t_{66}=$ 3.69; $P=0.001$; post hoc $t$-test, FDR corrected).

\section{Similarities and Differences Between Correlation Patterns are not Driven by Short-Term Changes in Resting-State Activations}

It is also important to consider the effects of systematic changes in resting-state patterns due to the visual stimuli or

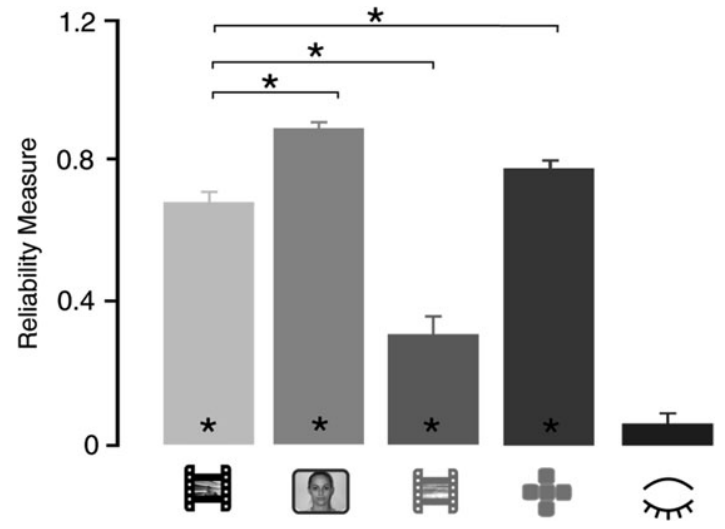

Figure 5. Response reliability. The bars show the mean response reliability between runs in the visual conditions (movie, categories 1-back, scrambled movie, and shape recognition) and during rest with EC. The reliability measure was calculated for each condition according to the symmetry of the cross-run matrices. Free viewing of a naturalistic movie showed significant reliability between runs; however, reliability was significantly lower than for the categories 1-back or shape recognition tasks. This result rules out the possibility that response reliability underlies the stronger similarity between the resting-state and movie-driven patterns. Error bars denote the standard error of the mean $( \pm \mathrm{SEM}){ }^{*} \mathrm{P}_{\mathrm{FDR}}<0.05$.

fatigue. To evaluate this issue, we analyzed the connectivity patterns for the first resting-state run (before watching the movie) and the second resting-state run (after watching the movie) separately. There was greater similarity between the postmovie resting state and the movie than between the premovie resting state and the movie (paired-sample t-test, $t_{14}=2.58, P=$ 0.02). Nevertheless, when we considered only the first restingstate run, which was performed before any other experimental condition, the movie-generated pattern still showed greater similarity to this first resting-state scan than to any of the other visual conditions $\left(F_{4,39}=31.7, P=0.0001\right.$; movie vs. categories 1-back, $t_{52}=2.366, P=0.03$; movie vs. shape recognition task, $t_{54}=4.909, P<0.0001 ;$ movie vs. scrambled movie, $t_{52}=2.973$, $P=0.0089$, FDR corrected). Since the order of the other conditions was counterbalanced across participants, this result rules out stimulus-driven changes in resting-state patterns as the main explanation of our results.

In order to probe for the effect of fatigue as an explanation for the increase in correlations in the postmovie resting-state run, we performed our main analysis again while taking only the first half of each task run or the second half. We hypothesized that if fatigue affected the results, we would find higher correlations between rest and the other conditions when taking into account the second half of the task runs (similarly to the effect we found when comparing the first rest and the second rest). Repeated-measures two-way mixed ANOVA on the correlations with the first resting-state run, with the factors visual condition (five levels: movie, categories 1-back, scrambled movie, shape recognition task, and rest) and run segment (two levels: first half and second half), confirmed a main effect of condition $\left(F_{4118}=17.45, P=0.001\right)$, but no interaction between condition and run segment $\left(F_{4118}=1.64, P=\right.$ 0.17). Importantly, there was no significant main effect of the run segment $\left(F_{1118}=3.12, P=0.08\right)$. This result argues against the confounding effect of drowsiness as the main explanation for the difference between the first and second rest runs, because fatigue would presumably increase during the latter phase of each run (i.e., the second segment). 


\section{Pattern Similarity does not Depend on the Specific Resting-State Condition}

In this study, we compared EC resting-state patterns with various visually evoked patterns. However, several studies have shown that ongoing spontaneous activity is modulated by the status of the participant, e.g., EC vs. EO, and, more specifically, that the EC condition increases the variability of the oxygen extraction fraction (cf. review of Gusnard and Raichle 2001). Thus, our results might be specific to the EC condition. To examine this possibility, 12 participants underwent two resting-state runs with EO and fixation, and we compared the EO resting-state and visually evoked patterns. All visual conditions correlated significantly with the resting condition. However, the resting condition was significantly more correlated with the naturalistic movie condition than the scrambled-movie condition or the shape recognition task (Fig. 6A). There were no significant differences in correlation strength between the resting and the movie conditions versus the resting condition and the categories 1-back task. This effect might be due to the small sample size or to the presence of a fixation point both in the EO resting condition and in the categories 1-back task, which might have increased their similarity due to a purely common visual stimulus (movie vs. scrambled movie, $t_{39}=4.2, P=0.0002$; movie vs. shape recognition task, $t_{40}=4.6, P$ $=0.0001$; post hoc $\mathrm{t}$-test, FDR corrected).
Response reliability across visual conditions in this subgroup of participants is shown in Figure $6 \mathrm{~B}$.

In addition, we directly tested the difference between resting with EO and resting with EC in the correlation patterns across all visual conditions. Figure $6 \mathrm{C}$ shows the mean correlation between the within-run resting-state pattern with EC and EO and the cross-run visual patterns. Paired t-tests for each condition showed no significant difference between rest with EC and rest with EO.

\section{Visual Conditions Contribution to the Model Selection}

To identify voxels located in higher order visual areas and avoid a potential bias toward one condition over the others, we selected the voxels based on the cross-run correlation of all visual conditions rather than just one condition. However, it is possible that the movie stimulus, with its rich and multidimensional content, might have driven activity and correlations across cortical regions more than the other, more artificial, stimuli. To assess the contribution of each visual condition to the voxel selection, we compared the voxel-by-voxel cross-run correlation map within each visual condition with the voxel-byvoxel cross-run correlation map calculated concatenating all the visual conditions (see Fig. 7). We found a significant difference between the visual conditions (one-way mixed ANOVA,
A
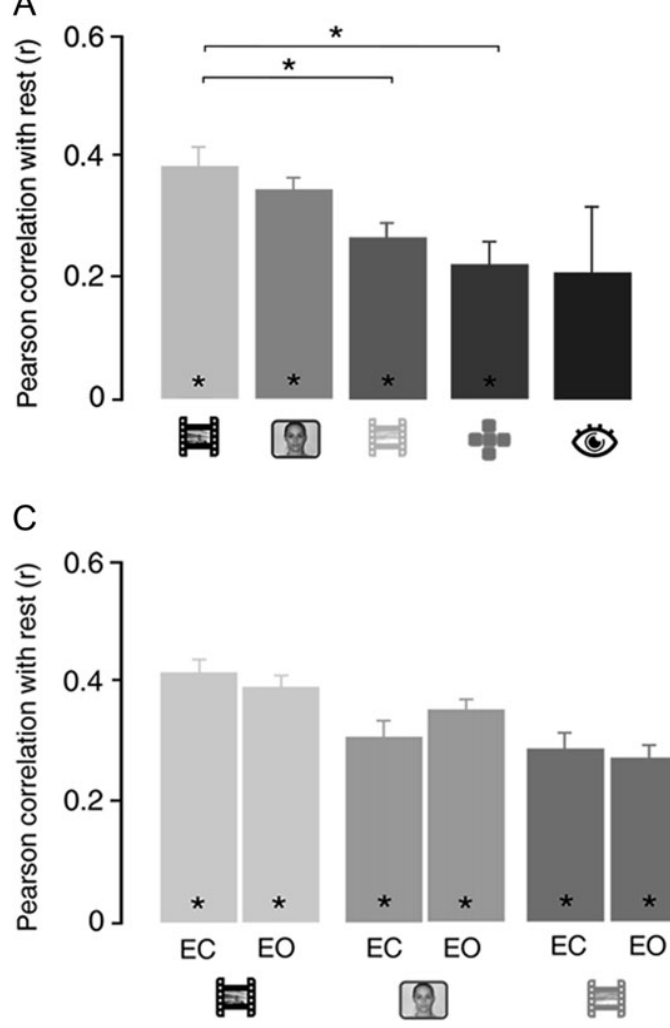

B

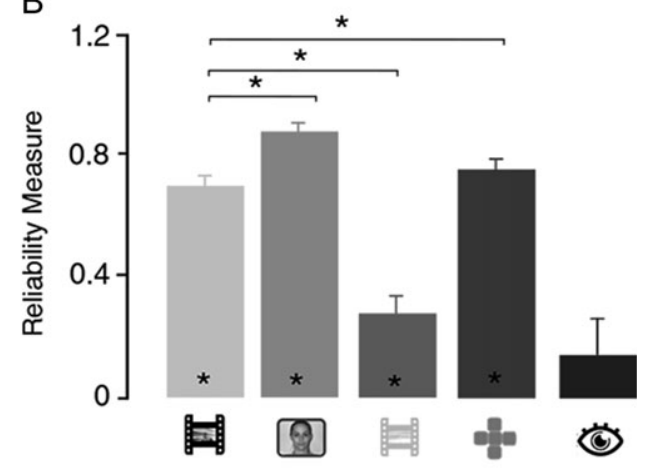

Figure 6. Correlation between EO resting-state pattern and visual conditions patterns. (A) The graph bars show the mean correlation between the within-run restingstate pattern with EO and the cross-run visual patterns (movie, $N=12$; categories 1-back, $N=12$; scrambled movie, $N=10$; shape recognition, $N=9$ ). All visual conditions showed a significant correlation to resting state, but the movie showed significantly higher correlation compared with the scrambled movie and shape recognition task. (B) Response reliability. The graph bars show the mean response reliability between runs in the visual conditions and during rest EO. The reliability measure was calculated for each condition using the symmetry of the cross-run matrices. Free-viewing movie showed a significant reliability between runs, however, it was significantly lower than the reliability in categories 1-back and shape recognition task. This result rules out the possibility that response reliability underlays the higher similarity between the resting-state and movie-driven patterns. (C) The graph bars show the mean correlation between the within-run resting-state pattern with EC and EO and the cross-run visual patterns. Error bars denote standard error of the mean $( \pm \mathrm{SEM}){ }^{*} \mathrm{P}<0.05$. 
$\left.F_{3,39}=69.01, P<0.0001\right)$. In particular, we found that categories 1-back task was more strongly correlated to the concatenated map than the other conditions including the movie condition (categories 1-back task vs. movie, $t_{38}=-2.842, P=0.0086$; categories 1-back task vs. shape recognition task, $t_{40}=-10.561, P<$ 0.001 ; categories 1 -back task vs. scrambled movie, $t_{39}=-11.989$, $P<0.001)$, and that the movie condition was more correlated than shape recognition task $\left(t_{40}=7.897, P<0.0001\right)$ and scrambled movie $\left(t_{39}=9.201, P<0.0001\right)$.

Additionally, the cross-run correlation maps showed that the activated voxels for each visual condition were mostly confined to the visual cortex.

\section{Pattern Similarity Across the Whole Cortex}

To investigate if resting-state patterns most strongly resembled naturalistic movie-induced patterns across the entire cortex (not restricted to visual areas), we explored the similarity between the resting and visual conditions with a searchlight approach (Kriegeskorte et al., 2006) across the whole cortex (see Methods). Figure 8 shows the average correlation between rest with EC and each of the visual conditions, constructed by averaging all the individual maps. Each map shows the similarity between the cross-run pattern and the rest pattern around each vertex. Highly correlated vertexes denote high similarity between the resting and the visual conditions in the area around the vertex. For all the visual conditions, the correlation patterns were extensive bilaterally in the visual cortex; however, movie and categories 1-back task showed a more widely distributed correlation pattern also spanning some regions outside of the visual cortex. In particular, in the movie condition, high correlations extended bilaterally in the superior temporal sulcus (STS), lateral sulcus (LS), precuneus (Precun), and intraparietal sulcus (IPS) (mostly in the right hemisphere). In contrast, both the scrambled movie and the shape recognition task produced a more localized pattern, restricted to the visual cortex.

We next calculated the correlation between the cross-run correlations for each visual condition and the within-run correlations during rest for all the activated voxels during the visual conditions (i.e., voxels with a minimum self-correlation of 0.25 , see Methods "Selection of Voxels for Pattern Extraction"). Overall, the pattern of results was similar to the one obtained in the higher order visual areas (Supplementary Fig. 3). We assessed the similarity between the visual condition and the rest, by performing a repeated-measures one-way mixed ANOVA. All visual conditions showed a significant correlation with the resting-state pattern (smallest $t_{12}=35.72, P=0.0001$ ). However, correlations between the resting and movie-viewing conditions were significantly higher than between the resting and shape recognition task and scrambled movie (movie vs. categories 1-back, $t_{52}=2, P=0.05$; movie vs. scrambled movie,

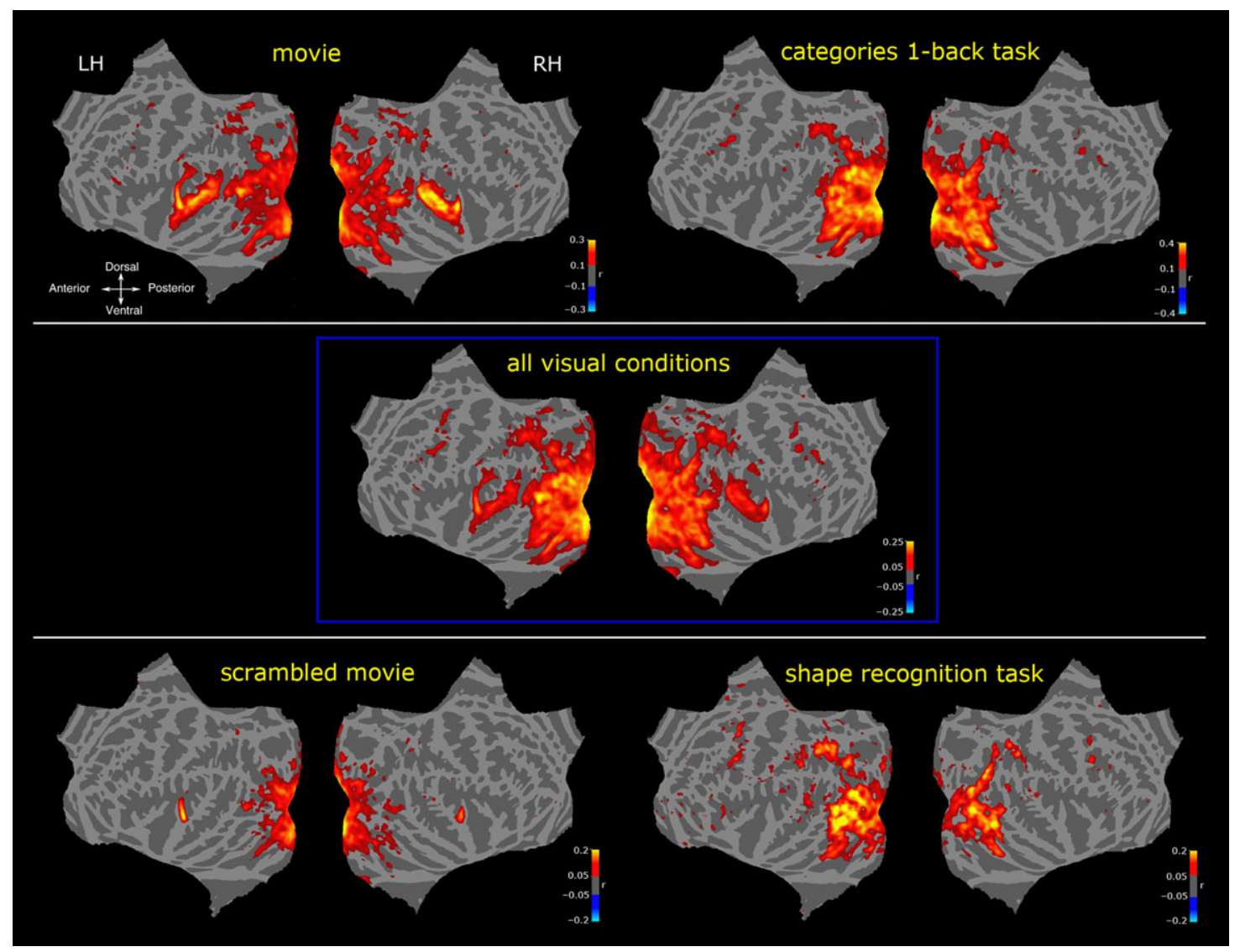

Figure 7. Voxel-by-voxel cross-run maps for each visual condition and for the concatenated conditions. The left and right hemispheres of an average surface are overlaid with voxel-by-voxel cross-run maps for the visual conditions: movie, categories 1-back task, scrambled movie, shape recognition task, and the concatenation of all the visual condition (central panel). Maps were averaged across subjects and projected onto the FreeSurfer fsaverage flat cortical surface (see Methods). Thresholds were chosen in order to equalize the number of voxels, thus to emphasize the similarity of correlation patterns across the maps rather than the differences in their strength (for display purposes only). 


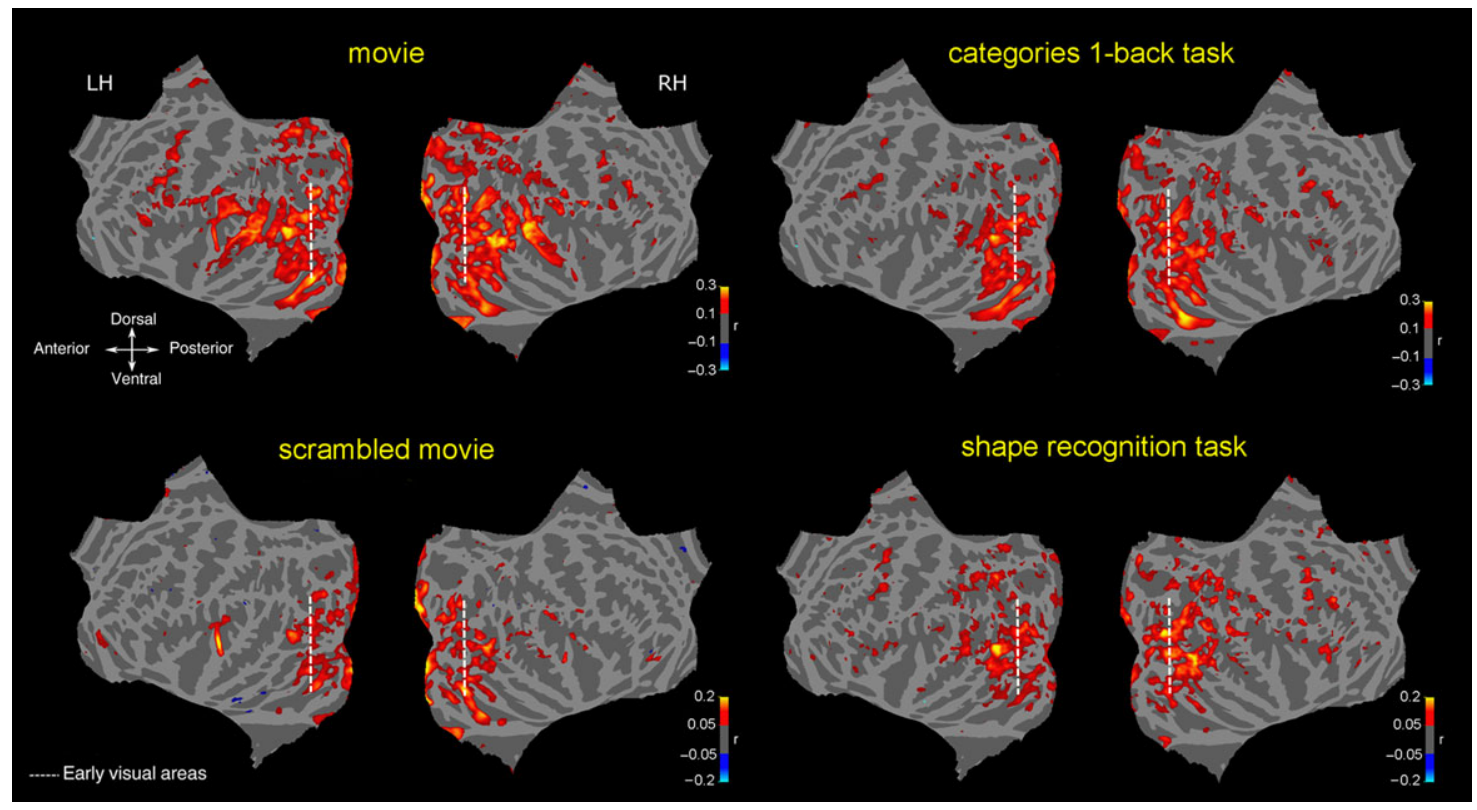

Figure 8. Group searchlight task-rest correlation maps. The left and right hemispheres are overlaid with a searchlight task-rest correlation group map (see Methods). The correlation coefficients were thresholded by a group-level one-sample t-test against zero (a Fisher transform of the coefficients ensured normality). Only vertices with $\mathrm{P}<0.05$ are shown. Maps were overlaid on the Freesurfer fsaverage flat cortical surface with 3 mm FWHM of surface smoothing and cluster correction for multiple comparisons at 0.01 (Hagler et al., 2006).

$t_{52}=2.67, P=0.01 ;$ movie vs. shape recognition, $t_{53}=4.62, P<$ 0.0001 ; post hoc t-test, FDR corrected).

\section{Discussion}

A substantial gap in our understanding of the neuronal mechanisms underlying human visual cortex function stems from the fact that most neurocognitive studies use highly artificial stimuli that are far removed from true ecological vision. Thus, a major concern in interpreting the vast set of findings derived from human visual cortex research is that they may be specific to artificial laboratory conditions and will fail to generalize to real-world conditions (Felsen and Dan 2005). It has been shown that naturalistic stimuli, with their rich spatiotemporal content, are more effective in driving neuronal activity (Touryan et al., 2005) and can elicit a unique neural response compared to synthetic stimuli (Felsen and Dan 2005). In addition, naturalistic stimuli can better reveal the neural response diversity and thus are more suitable for studying high-level sensory areas (Park et al., 2017).

Here, combining naturalistic stimuli and functional connectivity methods, we examined the hypothesis that restingstate fluctuations may provide information to bridge this gap. Specifically, we considered the prediction that cortical coactivations under conditions that more closely simulate real-life situations will be more similar to resting-state patterns than equally effective cortical activations driven by artificial stimuli rarely experienced in daily life. While movies are of course an imperfect equivalent for true ecological sensory stimulation, this condition allows participants to engage in free viewing of rich, naturalistic, multimodal and multidimensional dynamic stimuli (Hasson et al., 2004; Vanderwal et al., 2015). This cognitive engagement increases the reliability compared with resting-state measures and overcomes the potential negative effect of repeated viewings (Wang et al., 2017). Moreover, it has been shown that naturalistic movies allow to detect idiosyncratic functional connectivity patterns (Vanderwal et al., 2017).

Regarding the source of the similarity, in the introduction we proposed two models. According to one model, spontaneous patterns may reflect primarily the optical statistics of the natural world (Fiser et al., 2010); according to the second model, spontaneous patterns may reflect network coactivation statistics (Harmelech and Malach 2013). In particular, in the framework of the Bayesian probability theory, the two models differ in the content of the prior distribution. While the first model concerns the predictive statistics of the external environment (with a possible link to expectations, attention, etc.), the second model highlights the predictive statistics of the proper networks coactivations. It is a wellestablished phenomenon that in early visual cortex the network activations are retinotopically related to the low-level features of the optical stimuli. However, this retinotopic link makes it difficult to separate the role of optical from nonoptical factors in explaining the relation between spontaneous and evoked patterns. Importantly, this relation is significantly diminished in the higher order visual cortex. Although recent studies have shown that higher order visual areas are driven by the optical statistics of the world to some degree (Larsson and Heeger 2006; Kay et al., 2015; Uyar et al., 2016; Arcaro and Livingstone 2017; Kay and Yeatman 2017), these retinotopic inputs are minor and higher order visual areas are mainly driven by categorical and perceptual high-order gestalt (GrillSpector and Malach 2004; Fisch et al., 2009; Golan et al., 2016, 2017). A large body of research on neuropsychological patients has further supported this distinction in demonstrating that lesions in the higher order visual cortex may be associated with recognition and visuospatial deficits that involve all the visual field such as visual agnosia, body form agnosia, prosopagnosia, akinetopsia, and achromatopsia. Conversely, a lesion in the early visual areas is mainly associated with a retinotopic cortical representation deficit, a result further 
supported more recently by intracranial recordings (Winawer and Parvizi 2016). Thus, given this weakened relation between low-level optical statistics and functional specialization, higher order visual areas represent a good testing ground for the two models.

To test our prediction, we devised control stimuli that were effective in activating the tested brain regions, while creating a unique correlation pattern that was comparable between conditions. Moreover, intrasubject and inter-subject correlations in cortical activity during free movie and scrambled-movie viewing showed that whole-brain activations are mostly related to the visual cortex (Lu et al., 2016); thus suggesting that confining the analysis to the visual cortex can avoid the increase of possible sources of noise. The high-order visual cortex provided a good test for our hypotheses, while enabling the use of a set of control stimuli that prove the advantage of movie over other, more artificial, stimuli.

It could be argued that the higher correlation between categories 1-back task and the concatenation of all visual conditions might have biased the voxels selection and, therefore, the connectivity patterns. Consequently, one would expect an enhancement in the similarity between the categories 1-back condition and the resting-state patterns. However, our results on the concatenated visual conditions showed that despite such potential enhancement, the movie-induced patterns still showed significantly higher similarity to the resting-state patterns compared to the categories 1-back task.

Our results, showing a higher similarity between spontaneous and naturalistic evoked patterns, provide support to the hypothesis of a general principle of link between resting-state and habitual naturalistic neuronal coactivations. While previous research has revealed a robust and specific set of restingstate functional connections in various category-selective areas (Stevens et al., 2009, 2015; Hutchison et al., 2014; O'Neil et al., 2014; Chen et al. 2017), our study extends this research by showing that a significant component of this resting-state connectivity can be explained by the nature of cortical coactivations during movie viewing. Most importantly, our analysis focused specifically on the sensory-driven component of the responses, isolated from intrinsic resting-state activity by mapping correlations across repeated presentations of visual conditions. Previous studies, which either combined (Betti et al., 2013; Marussich et al., 2017) or separated (Kim et al., 2017) the effect of the sensory-driven and the intrinsic components, have addressed the complementary question about differences between functional connectivity patterns during movie viewing and rest.

Our approach, which relies on cross-run correlations to isolate sensory-driven patterns, is likely to provide only the lower bound of the true strength of activation patterns due to adaptation effects, attentional shifts, and eye movement variability across repeated runs. These effects may decrease the reproducibility of activations across movies, adding noise to the correlation patterns. The movie reliability index reflected this issue, which was lower than reliability indices for the more artificial but better-controlled categories 1-back and shape recognition tasks, although with a comparable or higher number of subjects. Remarkably, despite this lower reliability, the sensorydriven pattern emerging from the movie condition more strongly resembled the resting-state pattern than any other condition. The scrambled-movie condition presumably activated the category-selective cortex only weakly and hence showed a lower reliability index (Fig. 5). Similarly, previous results have reported that computer-generated animations comprised of abstract shapes with a slow and continuous motion (inscapes) showed a lower reliability index compared to movies (O'Connor et al., 2017).

It could be argued that our results were specific to the type of rest condition used for comparison with the visual conditions. It has been shown that different rest conditions are associated with different brain patterns (Marx et al., 2003, 2004; Hüfner et al., 2009; Jao et al., 2013; Xu et al., 2014; Zhang et al., 2015) and that the choice of rest condition is fundamental for estimating the change from baseline and to taskinduced BOLD activity (Gusnard and Raichle 2001). However, we found greater similarity between movie-induced patterns (compared with the other visual conditions) and rest with both EC and EO. These results indicate that the type of rest condition was not a critical factor in explaining the similarity of the patterns. However, although we did not find a significant difference between the conditions rest EO and rest EC, it is possible that because of the limited sample size we failed to detect a significant effect. In this regard, a potential limitation of this study is the small sample size. While some studies have pointed out that statistically significant results with small sample sizes indicate a stronger effect than the equivalent studies with a larger sample (Friston 2012), some others have shown the potential limitations of using small samples (Lindquist et al., 2013).

Another possible confound involves changes in resting-state connectivity induced by the tasks or movie viewing. These effects may have biased our results since half of the restingstate data were collected after movie viewing and the other half before. In fact, several studies have shown task-induced changes in resting-state functional connectivity lasting for hours or even days after the activation (Albert et al., 2009; Lewis et al., 2009; Stevens et al., 2009; Tambini et al., 2010; Tailby et al., 2015). Consistent with these previous findings, we found that the resting-state patterns generated by the second resting-state run (after movie viewing) were more similar to the movie than the first resting-state run (before movie viewing). However, even when only the first resting-state run was included in the analysis, (before movie viewing), the restingstate patterns remained more similar to the patterns associated with movie viewing than to any other visual stimulation condition. Thus, we can rule out the possibility that the similarities between the resting-state and movie conditions were somehow driven by a trace following the movie activation. The preferential correlation of the resting-state pattern with the movie-driven pattern, found even before the movie was shown, supports the conclusion that the similarity was driven by the typical statistics of natural, real-life sensory stimulation, rather than by the unique set of stimuli presented in the specific movie used in our experiment.

Finally, we explored the effect of general fatigue caused by the repeated fMRI scanning by running a split-half within-run analysis on the correlation matrices. However, we found no difference between the first and second half of each condition run when correlated to rest.

An important question concerns the mechanism that may embed the statistics of network activation in the spontaneous patterns. As we have proposed previously (Harmelech and Malach 2013), Hebbian learning offers a plausible and attractively simple mechanism. Thus, if we take higher order visual representations as an example, the model proposes that during daily life, networks that habitually coactivate will strengthen their functional connections due to a Hebbian learning process. When visual input is blocked during the resting mode, it is assumed that these networks are driven by inherent stochastic 
neuronal noise. In the absence of functional connectivity, this noise will be reflected in random, unstructured activation patterns (similar to "snow" on a television screen). However, the presence of synaptic connectivity among neurons imposes structure on this random pattern because the interneuronal connections tend to support spontaneously emerging neuronal coactivations. A physical metaphor for this phenomenon is the tendency of randomly oscillating pendula to gradually enter into sync due to subtle interactions. Furthermore, the more often a pair of cortical sites is coactivated, the stronger the resultant functional connectivity between them, leading to more highly correlated activation patterns that emerge during rest. Therefore, the spontaneous pattern revealed during rest can be envisioned as a sort of "archive" of the prior, habitual coactivation patterns that occur in the cortex over the course of an individual's ecological life.

Generating correlation patterns across all activated voxels in the cortex, we explored the similarity between rest and the visual conditions across the entire cortex (outside early retinotopic areas). We found that the similarity between movie and rest was higher than the similarity between rest and the other control visual conditions except for categories 1-back task (Supplementary Fig. 3). The lack of a significant difference between movie and categories 1-back task might be due to the limited sample size.

Mapping the relationship between rest and the visual conditions using the searchlight approach revealed that the similarity pattern between movie and rest extended beyond the visual cortex (Fig. 8). In particular, the extensive bilateral pattern in the movie condition that we found in the STS was presumably correlated to biological motion, which represents one of the strongest visual features during movie viewing (Russ and Leopold 2015; Goldberg et al., 2016), and to the processing of audiovisual social features during naturalistic stimulation (Lahnakoski et al., 2012). Moreover, in contrast with the other visual conditions, in the movie condition, the correlation patterns extended bilaterally in the precuneus. Interestingly, this multi-functional region has been associated with watching social interaction during realistic movie clips viewing (Iacoboni et al., 2004). The searchlight analysis also highlighted an important feature of our analysis approach: because the analysis was based on cross-run functional connectivity, only areas that were activated by the task showed an informative connectivity pattern. Therefore, our choice of the artificial stimulus conditions was targeted at activating specific networks of interest (in our case, the visual system). Future research using control stimuli activating, for example, the auditory cortex could be performed to test the generalization of our findings to other modalities.

The specific elements of the movie driving the similarity between resting-state and movie-induced patterns are difficult to assess. Indeed, we have found that neither the motion component of the movie (scrambled movie) nor the static naturalistic images (categories 1-back task) accounted for the full richness of spontaneous patterns as captured in the naturalistic movie. Features absent from the artificial stimuli but present in the movie (and in real life) may underlie the effect, such as a semantic narrative (Zacks et al., 2011), the events structure (Baldassano et al., 2017), the complexity of the scene (in particular the simultaneous presence of several object categories, compared with the more isolated object presentation in the control conditions), fine-grained temporal resolution (Spiers and Maguire 2007), simultaneous audiovisual stimulation, and biological motion (Russ and Leopold 2015). More extensive studies isolating these dimensions (and others) are needed to precisely identify the sources of the similarity between patterns associated with naturalistic stimuli and the resting state and to assess how this similarity changes across the development of each individual (Berkes et al., 2011) as a result of reshaping driven by coupling with the dynamic regularities of the environment.

\section{Supplementary Material}

Supplementary material is available at Cerebral Cortex online.

\section{Funding}

The CIFAR Azrieli Program on Brain, Mind, and Consciousness, ICORE funding to R.M., the IRCCS NEUROMED, and the Italian Ministry of Education, University, and Research.

\section{Notes}

The authors would like to thank Fanny Attar and Nahum Stern, for assistance with fMRI scanning, Michal Ramot for movie editing, and Nir Bergman for copyright permission. Conflict of Interest: The authors declare no competing financial interests.

\section{References}

Albert NB, Robertson EM, Miall RC. 2009. The resting human brain and motor learning. Curr Biol. 19(12):1023-1027.

Arcaro MJ, Livingstone MS. 2017. A hierarchical, retinotopic proto-organization of the primate visual system at birth. Elife. 6:e26196.

Arieli A, Sterkin A, Grinvald A, Aertsen AD. 1996. Dynamics of ongoing activity: explanation of the large variability in evoked cortical responses. Science. 273(5283):1868.

Baldassano C, Chen J, Zadbood A, Pillow JW, Hasson U, Norman KA. 2017. Discovering event structure in continuous narrative perception and memory. Neuron. 95(3):709-721.e5. doi:10.1016/j.neuron.2017.06.041.

Bates D, Mächler M, Bolker B, Walker S 2014. Fitting linear mixed-effects models using lme4. arXiv preprint arXiv: 1406.5823.

Behrmann M, Plaut DC. 2013. Distributed circuits, not circumscribed centers, mediate visual recognition. Trends Cogn Sci. 17(5):210-219.

Berkes P, Orbán G, Lengyel M, Fiser J. 2011. Spontaneous cortical activity reveals hallmarks of an optimal internal model of the environment. Science. 331(6013):83-87.

Betti V, Della Penna S, de Pasquale F, Mantini D, Marzetti L, Romani GL, Corbetta M. 2013. Natural scenes viewing alters the dynamics of functional connectivity in the human brain. Neuron. 79(4):782-797.

Biswal B, Zerrin Yetkin F, Haughton VM, Hyde JS. 1995. Functional connectivity in the motor cortex of resting human brain using echo-planar mri. Magn Reson Med. 34(4):537-541.

Brodeur MB, Dionne-Dostie E, Montreuil T, Lepage M. 2010. The Bank of Standardized Stimuli (BOSS), a new set of 480 normative photos of objects to be used as visual stimuli in cognitive research. PLoS One. 5(5):e10773.

Chen Q Garcea FE, Almeida J, Mahon BZ. 2017. Connectivitybased constraints on category-specificity in the ventral object processing pathway. Neuropsychologia. 105:184-196.

Ciric R, Wolf DH, Power JD, Roalf DR, Baum GL, Ruparel K, Shinohara RT, Elliott MA, Eickhoff SB, Davatzikos C, et al. 
2017. Benchmarking of participant-level confound regression strategies for the control of motion artifact in studies of functional connectivity. Neuroimage. 154:174-187.

Cole MW, Ito T, Bassett DS, Schultz DH. 2016. Activity flow over resting-state networks shapes cognitive task activations. Nat Neurosci. 19(12):1718-1726.

Dale AM, Fischl B, Sereno MI. 1999. Cortical surface-based analysis: I. Segmentation and surface reconstruction. Neuroimage. 9(2):179-194.

Downing PE, Jiang Y, Shuman M, Kanwisher N. 2001. A cortical area selective for visual processing of the human body. Science. 293(5539):2470-2473.

Ellis D 2010. Time-domain scrambling of audio signals in Matlab.

Epstein R, Kanwisher N. 1998. A cortical representation of the local visual environment. Nature. 392(6676):598-601.

Felsen G, Dan Y. 2005. A natural approach to studying vision. Nat Neurosci. 8(12):1643.

Finn ES, Shen X, Scheinost D, Rosenberg MD, Huang J, Chun MM, Papademetris X, Constable RT. 2015. Functional connectome fingerprinting: identifying individuals using patterns of brain connectivity. Nat Neurosci. 18(11):1664-1671.

Fisch L, Privman E, Ramot M, Harel M, Nir Y, Kipervasser S, Andelman F, Neufeld MY, Kramer U, Fried I, et al. 2009. Neural "ignition": enhanced activation linked to perceptual awareness in human ventral stream visual cortex. Neuron. 64(4):562-574.

Fischl B, Salat DH, Busa E, Albert M, Dieterich M, Haselgrove C, van der Kouwe A, Killiany R, Kennedy D, Klaveness S, et al. 2002. Whole brain segmentation: automated labeling of neuroanatomical structures in the human brain. Neuron. 33 (3):341-355.

Fiser J, Berkes P, Orbán G, Lengyel M. 2010. Statistically optimal perception and learning: from behavior to neural representations. Trends Cogn Sci. 14(3):119-130.

Fox MD, Corbetta M, Snyder AZ, Vincent JL, Raichle ME. 2006. Spontaneous neuronal activity distinguishes human dorsal and ventral attention systems. Proc Natl Acad Sci. 103(26): 10046-10051.

Fox MD, Raichle ME. 2007. Spontaneous fluctuations in brain activity observed with functional magnetic resonance imaging. Nat Rev Neurosci. 8(9):700-711.

Fox MD, Zhang D, Snyder AZ, Raichle ME. 2009. The global signal and observed anticorrelated resting state brain networks. J Neurophysiol. 101(6):3270-3283.

Friston K. 2012. Ten ironic rules for non-statistical reviewers. Neuroimage. 61(4):1300-1310.

Froudarakis E, Berens P, Ecker AS, Cotton RJ, Sinz FH, Yatsenko D, Saggau P, Betge M, Tolias AS. 2014. Population code in mouse V1 facilitates readout of natural scenes through increased sparseness. Nat Neurosci. 17(6):851-857.

Geerligs L, Rubinov M, Henson RN. 2015. State and trait components of functional connectivity: individual differences vary with mental state. J Neurosci. 35(41):13949-13961.

Golan T, Davidesco I, Meshulam M, Groppe DM, Mégevand P, Yeagle EM, Goldfinger MS, Harel M, Melloni L, Schroeder CE, et al. 2016. Human intracranial recordings link suppressed transients rather than'filling-in'to perceptual continuity across blinks. Elife. 5:e17243.

Golan T, Davidesco I, Meshulam M, Groppe DM, Mégevand P, Yeagle EM, Goldfinger MS, Harel M, Melloni L, Schroeder CE, et al. 2017. Increasing suppression of saccade-related transients along the human visual hierarchy. eLife, 6, e27819.
Goldberg H, Hart Y, Mayo A, Alon U, Malach R. 2016. Neuronal components of evaluating the human 1 origin of abstract shapes. bioRxiv:085902.

Golland Y, Bentin S, Gelbard H, Benjamini Y, Heller R, Nir Y, Hsson U, Malach R. 2007. Extrinsic and intrinsic systems in the posterior cortex of the human brain revealed during natural sensory stimulation. Cereb Cortex. 17(4): 766-777.

Greicius MD, Menon V. 2004. Default-mode activity during a passive sensory task: uncoupled from deactivation but impacting activation. J Cogn Neurosci. 16(9):1484-1492.

Greve DN, Fischl B. 2009. Accurate and robust brain image alignment using boundary-based registration. Neuroimage. 48(1):63-72.

Grill-Spector K, Malach R. 2004. The human visual cortex. Annu Rev Neurosci. 27:649-677.

Gusnard DA, Raichle ME. 2001. Searching for a baseline: functional imaging and the resting human brain. Nat Rev Neurosci. 2(10):685-694.

Hagler DJ Jr., Saygin AP, Sereno MI. 2006. Smoothing and cluster thresholding for cortical surface-based group analysis of fMRI data. Neuroimage. 33(4):1093-1103.

Hahamy A, Sotiropoulos SN, Slater DH, Malach R, JohansenBerg H, Makin TR. 2015. Normalisation of brain connectivity through compensatory behaviour, despite congenital hand absence. Elife. 4:e04605.

Harmelech T, Malach R. 2013. Neurocognitive biases and the patterns of spontaneous correlations in the human cortex. Trends Cogn Sci. 17(12):606-615.

Harmelech T, Preminger S, Wertman E, Malach R. 2013. The day-after effect: long term, Hebbian-like restructuring of resting-state fMRI patterns induced by a single epoch of cortical activation. J Neurosci. 33(22):9488-9497.

Hasson U, Malach R, Heeger DJ. 2010. Reliability of cortical activity during natural stimulation. Trends Cogn Sci. 14(1): $40-48$.

Hasson U, Nir Y, Levy I, Fuhrmann G, Malach R. 2004. Intersubject synchronization of cortical activity during natural vision. Science. 303(5664):1634-1640.

Hüfner K, Stephan T, Flanagin VL, Deutschländer A, Stein A, Kalla R, Dera T, Fesl G, Jahn K, Strupp M, et al. 2009. Differential effects of eyes open or closed in darkness on brain activation patterns in blind subjects. Neurosci Lett. 466(1):30-34.

Hutchison RM, Culham JC, Everling S, Flanagan JR, Gallivan JP. 2014. Distinct and distributed functional connectivity patterns across cortex reflect the domain-specific constraints of object, face, scene, body, and tool category-selective modules in the ventral visual pathway. Neuroimage. 96: 216-236.

Iacoboni M, Lieberman MD, Knowlton BJ, Molnar-Szakacs I, Moritz M, Throop CJ, Fiske AP. 2004. Watching social interactions produces dorsomedial prefrontal and medial parietal BOLD fMRI signal increases compared to a resting baseline. Neuroimage. 21(3):1167-1173.

Jao T, Vértes PE, Alexander-Bloch AF, Tang IN, Yu YC, Chen JH, Bullmore ET. 2013. Volitional eyes opening perturbs brain dynamics and functional connectivity regardless of light input. Neuroimage. 69:21-34.

Jenkinson M, Bannister P, Brady M, Smith S. 2002. Improved optimization for the robust and accurate linear registration and motion correction of brain images. Neuroimage. 17(2): 825-841. 
Kanwisher N, McDermott J, Chun MM. 1997. The fusiform face area: a module in human extrastriate cortex specialized for face perception. J Neurosci. 17(11):4302-4311.

Kay KN, Weiner KS, Grill-Spector K. 2015. Attention reduces spatial uncertainty in human ventral temporal cortex. Curr Biol. 25(5):595-600.

Kay KN, Yeatman JD. 2017. Bottom-up and top-down computations in word-and face-selective cortex. Elife. 6:e22341.

Kenet T, Bibitchkov D, Tsodyks M, Grinvald A, Arieli A. 2003. Spontaneously emerging cortical representations of visual attributes. Nature. 425(6961):954-956.

Kim D, Kay K, Shulman GL, Corbetta M. 2017. A new modular brain organization of the BOLD signal during natural vision. Cereb Cortex. 28(9):3065-3081.

Kriegeskorte N, Goebel R, Bandettini P. 2006. Information-based functional brain mapping. Proc Natl Acad Sci USA. 103(10): 3863-3868.

Lahnakoski JM, Glerean E, Salmi J, Jääskeläinen IP, Sams M, Hari R, Nummenmaa L. 2012. Naturalistic FMRI mapping reveals superior temporal sulcus as the hub for the distributed brain network for social perception. Front Hum Neurosci. 6:233.

Larsson J, Heeger DJ. 2006. Two retinotopic visual areas in human lateral occipital cortex. J Neurosci. 26(51):13128-13142.

Levy I, Hasson U, Avidan G, Hendler T, Malach R. 2001. Centerperiphery organization of human object areas. Nat Neurosci. 4(5):533.

Lewis JW. 2006. Cortical networks related to human use of tools. Neuroscientist. 12(3):211-231.

Lewis CM, Baldassarre A, Committeri G, Romani GL, Corbetta M. 2009. Learning sculpts the spontaneous activity of the resting human brain. Proc Natl Acad Sci. 106(41):17558-17563.

Lindquist MA, Caffo B, Crainiceanu C. 2013. Ironing out the statistical wrinkles in "ten ironic rules". Neuroimage. 81: 499-502.

Lu KH, Hung SC, Wen H, Marussich L, Liu Z. 2016. Influences of high-level features, gaze, and scene transitions on the reliability of BOLD responses to natural movie stimuli. PloS one. 11(8):e0161797.

Mahon BZ, Caramazza A. 2011. What drives the organization of object knowledge in the brain? Trends Cogn Sci. 15(3): 97-103.

Makris N, Kaiser J, Haselgrove C, Seidman LJ, Biederman J, Boriel D, Valera EM, Papadimitriou GM, Fischl B, Caviness VS Jr., et al. 2006. Human cerebral cortex: a system for the integration of volume-and surface-based representations. Neuroimage. 33(1):139-153.

Marussich L, Lu KH, Wen H, Liu Z. 2017. Mapping white-matter functional organization at rest and during naturalistic visual perception. Neuroimage. 146:1128-1141.

Marx E, Deutschländer A, Stephan T, Dieterich M, Wiesmann M, Brandt T. 2004. Eyes open and eyes closed as rest conditions: impact on brain activation patterns. Neuroimage. 21 (4):1818-1824.

Marx E, Stephan T, Nolte A, Deutschländer A, Seelos KC, Dieterich M, Brandt T. 2003. Eye closure in darkness animates sensory systems. Neuroimage. 19(3):924-934.

Minagawa-Kawai Y, Van Der Lely H, Ramus F, Sato Y, Mazuka R, Dupoux E. 2010. Optical brain imaging reveals general auditory and language-specific processing in early infant development. Cereb Cortex. 21(2):254-261.

Minear M, Park DC. 2004. A lifespan database of adult facial stimuli. Behav Res Methods Instrum Comput. 36(4):630-633.
Noy L, Hart Y, Andrew N, Ramote O, Mayo AE, Alon U. 2012. A quantitative study of creative leaps. Proc. Third Int. Conf. Comput. Creativity, Dublin, pp 72-76.

O'Connor D, Potler NV, Kovacs M, Xu T, Ai L, Pellman J, Vanderwal T, Parra LC, Cohen S, Ghosh S, et al. 2017. The healthy brain network serial scanning initiative: a resource for evaluating inter-individual differences and their reliabilities across scan conditions and sessions. Gigascience. 6(2):1-14.

O’Neil EB, Hutchison RM, McLean DA, Köhler S. 2014. Restingstate fMRI reveals functional connectivity between faceselective perirhinal cortex and the fusiform face area related to face inversion. Neuroimage. 92:349-355.

Park SH, Russ BE, McMahon DB, Koyano KW, Berman RA, Leopold DA. 2017. Functional subpopulations of neurons in a macaque face patch revealed by single-unit fMRI mapping. Neuron. 95(4):971-981.

Power JD, Barnes KA, Snyder AZ, Schlaggar BL, Petersen SE. 2012. Spurious but systematic correlations in functional connectivity MRI networks arise from subject motion. Neuroimage. 59(3):2142-2154.

Power JD, Mitra A, Laumann TO, Snyder AZ, Schlaggar BL, Petersen SE. 2014. Methods to detect, characterize, and remove motion artifact in resting state fMRI. Neuroimage. 84:320-341.

Russ BE, Leopold DA. 2015. Functional MRI mapping of dynamic visual features during natural viewing in the macaque. Neuroimage. 109:84-94.

Sadaghiani S, Kleinschmidt A. 2013. Functional interactions between intrinsic brain activity and behavior. Neuroimage. 80:379-386.

Sereno MI, Dale AM, Reppas JB, Kwong KK, Belliveau JW, Brady TJ, Tootell RB. 1995. Borders of multiple visual areas in humans revealed by functional magnetic resonance imaging. Sci. 268(5212):889-893.

Shibata K, Watanabe T, Sasaki Y, Kawato M. 2011. Perceptual learning incepted by decoded fMRI neurofeedback without stimulus presentation. Science. 334(6061):1413-1415.

Siegel JS, Mitra A, Laumann TO, Seitzman BA, Raichle M, Corbetta M, Snyder AZ. 2016. Data quality influences observed links between functional connectivity and behavior. Cereb Cortex. 27(9):4492-4502.

Singmann H, Bolker B, Westfall J 2015. Afex: analysis of factorial experiments. R package version 0.13-145.

Smith SM, Fox PT, Miller KL, Glahn DC, Fox PM, Mackay CE, Filippini N, Watkins KE, Toro R, Laird AR, et al. 2009. Correspondence of the brain's functional architecture during activation and rest. Proc Natl Acad Sci. 106(31):13040-13045.

Snyder AZ, Raichle ME. 2012. A brief history of the resting state: the Washington University perspective. Neuroimage. 62(2):902-910.

Spiers HJ, Maguire EA. 2007. Decoding human brain activity during real-world experiences. Trends Cogn Sci. 11(8):356-365.

Stevens WD, Buckner RL, Schacter DL. 2010. Correlated lowfrequency BOLD fluctuations in the resting human brain are modulated by recent experience in category-preferential visual regions. Cereb Cortex. 20:1997-2006.

Stevens WD, Tessler MH, Peng CS, Martin A. 2015. Functional connectivity constrains the category-related organization of human ventral occipitotemporal cortex. Hum Brain Mapp. 36(6):2187-2206.

Tailby C, Masterton RA, Huang JY, Jackson GD, Abbott DF. 2015. Resting state functional connectivity changes induced by prior brain state are not network specific. Neuroimage. 106: 428-440. 
Tambini A, Ketz N, Davachi L. 2010. Enhanced brain correlations during rest are related to memory for recent experiences. Neuron. 65(2):280-290.

Tavor I, Jones OP, Mars RB, Smith SM, Behrens TE, Jbabdi S. 2016. Task-free MRI predicts individual differences in brain activity during task performance. Science. 352(6282):216-220.

Tong F, Nakayama K, Vaughan JT, Kanwisher N. 1998. Binocular rivalry and visual awareness in human extrastriate cortex. Neuron. 21(4):753-759.

Touryan J, Felsen G, Dan Y. 2005. Spatial structure of complex cell receptive fields measured with natural images. Neuron. 45(5):781-791.

Tsodyks M, Kenet T, Grinvald A, Arieli A. 1999. Linking spontaneous activity of single cortical neurons and the underlying functional architecture. Science. 286(5446):1943-1946.

Uyar F, Shomstein S, Greenberg AS, Behrmann M. 2016. Retinotopic information interacts with category selectivity in human ventral cortex. Neuropsychologia. 92:90-106.

Vanderwal T, Kelly C, Eilbott J, Mayes LC, Castellanos FX. 2015. Inscapes: a movie paradigm to improve compliance in functional magnetic resonance imaging. Neuroimage. 12:222-232.

Vanderwal T, Eilbott J, Finn ES, Craddock RC, Turnbull A, Castellanos FX. 2017. Individual differences in functional connectivity during naturalistic viewing conditions. Neuroimage. 157:521-530.

Wang D, Buckner RL, Fox MD, Holt DJ, Holmes AJ, Stoecklein S, Langs G, Pan R, Quan T, Li K, et al. 2015. Parcellating cortical functional networks in individuals. Nat Neurosci. 18:1853-1860.
Wang J, Ren Y, Hu X, Nguyen VT, Guo L, Han J, Guo CC. 2017. Test-retest reliability of functional connectivity networks during naturalistic fMRI paradigms. Hum Brain Mapp. 38(4): 2226-2241.

Wilf M, Strappini F, Golan T, Hahamy A, Harel M, Malach R. 2015. Spontaneously Emerging Patterns in Human Visual Cortex Reflect Responses to Naturalistic Sensory Stimuli. Cereb Cortex. 27:750-763.

Winawer J, Parvizi J. 2016. Linking electrical stimulation of human primary visual cortex, size of affected cortical area, neuronal responses, and subjective experience. Neuron. 92 (6):1213-1219.

Xu P, Huang R, Wang J, Van Dam NT, Xie T, Dong Z, Chen C, Gu R, Zang YF, He Y, et al. 2014. Different topological organization of human brain functional networks with eyes open versus eyes closed. Neuroimage. 90:246-255.

Zacks JM, Kurby CA, Eisenberg ML, Haroutunian N. 2011. Prediction error associated with the perceptual segmentation of naturalistic events. J Cogn Neurosci. 23(12): 4057-4066.

Zhang Y, Brady M, Smith S. 2001. Segmentation of brain MR images through a hidden Markov random field model and the expectation-maximization algorithm. IEEE Trans Med Imaging. 20(1):45-57.

Zhang D, Liang B, Wu X, Wang Z, Xu P, Chang S, Liu B, Liu M, Huang R. 2015. Directionality of large-scale resting-state brain networks during eyes open and eyes closed conditions. Front Hum Neurosci. 9:81. 